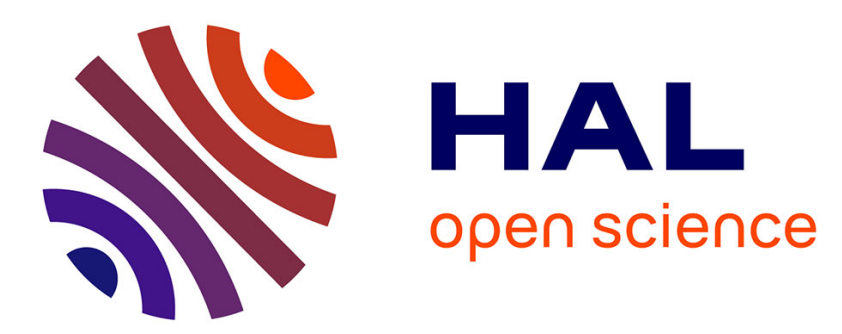

\title{
Les divinités d'origine indigène et iranienne sur le monnayage des cités du Pont sous les premiers Sévères
}

Julie Dalaison, Bernard Rémy

\section{To cite this version:}

Julie Dalaison, Bernard Rémy. Les divinités d'origine indigène et iranienne sur le monnayage des cités du Pont sous les premiers Sévères . Revue Numismatique, 2013, 170, pp.29-60. halshs-01429175

\section{HAL Id: halshs-01429175 \\ https://shs.hal.science/halshs-01429175}

Submitted on 10 Jan 2017

HAL is a multi-disciplinary open access archive for the deposit and dissemination of scientific research documents, whether they are published or not. The documents may come from teaching and research institutions in France or abroad, or from public or private research centers.
L'archive ouverte pluridisciplinaire HAL, est destinée au dépôt et à la diffusion de documents scientifiques de niveau recherche, publiés ou non, émanant des établissements d'enseignement et de recherche français ou étrangers, des laboratoires publics ou privés. 


\section{Julie DaLAison, Bernard RÉmY* \\ Les divinités d'origine indigène et iranienne sur
le monnayage des cités du Pont sous les premiers Sévères}

Résumé - C'est sans doute à la fin de 114, que les cités du Pont Galatique et Polémoniaque sont passées de la Galatie à la Cappadoce. Dans leur panthéon «monétaire», nous retrouvons des divinités d'origine indigène, iranienne et gréco-romaines. Sauf Sebastopolis, les cinq autres cités honorent sur leur monnayage une divinité d'origine indigène ou iranienne, propre à chaque cité. Leur survivance à l'époque sévérienne n'est pas liée à un quelconque regain d'intérêt pour un passé lointain, mais confirme l'importance de l'influence indigène et iranienne dans le Pont. Toutefois, ces divinités ont profondément évolué. Elles sont le résultat d'une histoire au cours de laquelle différentes «couches» se sont superposées.

Mots clés - Pont, Anahita, Mâ, Mèn, Mithra, Zeus Stratios.

Summary - It is probably at the end of 114 AD that the cities of Galatic and Polemoniac Pontus, previously belonging to Galatia, moved to Cappadocia. Among the pantheon of deities depicted on their coinage we find native Iranian and Graeco-Roman gods. Save for Sebastopolis, each of the other cities celebrated on its coins a native or Iranian deity of its own. The persistence of this practice during the Severan period is not linked to some renewed interest in a remote past, but rather confirms how important were local and Iranian influences in Pontus. Even so, the gods as depicted have evolved markedly, the result of a history in which different layers have superimposed themselves.

Keywords - Pontus, Anahita, Mâ, Mèn, Mithra, Zeus Stratios.

\section{Introduction}

C'est sans doute sous Trajan, dès la fin de $114^{1}$, que les cités du Pont, c'està-dire du Pont Galatique (Amaseia, Sebastopolis et Comana Pontica) et du Pont Polémoniaque (Polemonium, Cerazus, Trapezus, Neocaesarea, Zela et Sebasteia) sont passées de la Galatie à la Cappadoce, dont elles étaient le complément naturel. En effet, cette nouvelle affectation permettait au légat impérial consulaire de Cappadoce de contrôler le nord de l'Arménie et le sud du Caucase, deux régions montagneuses vitales pour la sécurité de la frontière orientale de l'empire romain.

\footnotetext{
* Julie Dalaison (Université Lumière Lyon 2, 86, rue Pasteur, 69365 Lyon Cedex 07) est maître de conférences d'histoire romaine à l'université Lyon 2 (CNRS, HiSoMA, Lyon) ; Bernard Rémy (Les Thermes, 73230 Saint-Jean-d'Arvey) est professeur émérite d'histoire romaine de l'université de Grenoble 2 (CRHIPA ; CNRS, Centre Camille Jullian, Aix-en-Provence). Merci à Nicole Belayche de ses précieuses remarques et à Isabelle Fauduet pour la traduction anglaise.

1. RÉmY 1986, p. 72-73. Pour la localisation des sites, voir la figure 1.
} 
Toutefois, nous n'avons pas de preuves formelles de ce transfert avant le règne d'Hadrien ${ }^{2}$, où le gouverneur de Cappadoce L. (?) Statorius Secundus apparaît dans un document épigraphique de Sebastopolis ${ }^{3}$ et sur des monnaies de Césarée de Cappadoce 4 .

Cette situation a perduré jusque sous le règne de Sévère Alexandre, où fut créée une province équestre du Pont réunissant dans la même entité administrative les cités du Pont Galatique et du Pont Polémoniaque. Elle est attestée par une inscription du territoire d'Amaseia ${ }^{5}$, gravée vers $233-235^{6}$, en l'honneur de Sévère Alexandre par Q. Faltonius Restitutianus, procurator et praeses prouinciae Ponti. Sous les deux premiers Sévères, les six cités pontiques qui ont alors frappé monnaie $\left(\right.$ Amaseia $^{7}$, Comana Pontica $^{8}$, Neocaesarea $^{9}$, Sebastopolis $^{10}$, Trapezus $^{11}$, Zela $^{12}$ ) relevaient donc de la province de Cappadoce.

Ces six cités appartenaient vraisemblablement au même koinon, celui du Pont. D'après Xavier Loriot ${ }^{13}$, il n'aurait existé qu'un seul koinon du Pont, et non deux koina. La thèse des deux koina distinguait un koinon du Pont occidental (avec pour capitale Heraclea Pontica ou Amastris) regroupant les cités de la partie pontique de la province de Pont-Bithynie et un koinon du Pont oriental - plus tardif (avec pour capitale Neocaesarea) rassemblant les cités du Pont Galatique et du Pont Polémoniaque. Xavier Loriot, reprenant en partie des arguments avancés par Jürgen Deininger ${ }^{14}$, réfute de façon convaincante cette théorie. Il rappelle que les deux prétendus koina sont désignés de la même manière et que les pièces

2. C'est probablement au même moment que les trois cités d'Abonoteichos-Ionopolis, Sinope et Amisos ont été transférées du Pont-Bithynie à la Galatie pour donner à cette province un accès à la mer Noire (RÉMY 1986, p. 90-93 ; LORIOT 2011, p. 283-284).

3. AE 1968, no 504 ; sur ce personnage, voir RÉMY 1989, no 167.

4. Sydenham 1933, p. 73, no 29 et Nony 1971, p. 37. Pour le règne de Trajan, nous avons seulement un témoignage a silentio dans la titulature de l'anonyme de CIL III, no 6819 qui porte le titre de legatus Augusti pro praetore prouinciae Galatiae, Phrygiae, Pisisidiae, Lycaoniae, Paphlagoniae (RÉmY 1989, no 109).

5. French 1986, p. 75 (AE 1986, no 653).

6. Voir Christol, LoRIOT 1986, p. 13-40.

7. En 205/206, au nom de Septime Sévère, Iulia Domna, Caracalla et Géta ; en 206/207, au nom de Septime Sévère, Iulia Domna, Caracalla (voir Dalaison 2008).

8. En 205/206, au nom de Septime Sévère, Iulia Domna, Caracalla et Géta (voir Amandry, RÉMY 1999).

9. En 204/205, au nom de Septime Sévère, Caracalla et Géta ; en 205/206, au nom de Septime Sévère, Iulia Domna, Caracalla et Géta (voir Çızmeli 2006).

10. En 205/206, au nom de Septime Sévère, Iulia Domna, Caracalla et Géta (voir AmANDRY, RÉMY 1998).

11. En 194/195, au nom de Septime Sévère et Iulia Domna ; en 198/199, au nom de Caracalla et Géta ; en 204/205, au nom de Septime Sévère ; en 216/217, au nom de Caracalla et Iulia Domna (voir WoJAn 2006).

12. En 205/206 et 206/207, au nom de Septime Sévère, Iulia Domna Caracalla et Géta (voir DALAISON, RÉmy, AMANDRY 2009).

13. LORIOT 2006, p. 528-532. Voir aussi ÇIZMELi 2006, p. 96-97 et 126.

14. DeININGER 1965, p. 64-66. 


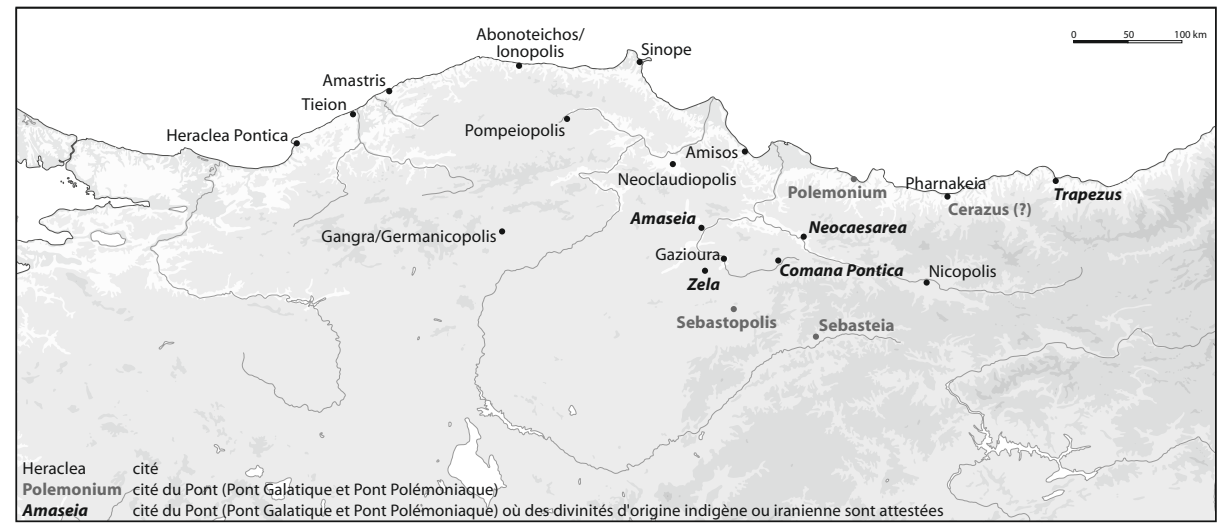

Figure 1 - Carte de localisation.

de Neocaesarea représentant six Tychès (censées faire référence aux six cités membres du koinon du Pont oriental) ne sont pas une preuve certaine, du fait de l'incertitude liée aux représentations iconographiques sur les monnaies ${ }^{15}$. Nous pouvons également ajouter, si l'on suppose que le chiffre six n'a pas été choisi au hasard, que ces six Tychès peuvent faire référence, soit aux six cités du district du Pont Polémoniaque (Polemonium, Cerazus, Trapezus, Neocaesarea, Zela et Sebasteia), soit aux six cités de la région géographique du Pontus Mediterraneus ou Mésogée (Neocaesarea, Amaseia, Comana Pontica, Zela, Sebastopolis et peut-être Sebasteia $)^{16}$. Xavier Loriot mentionne en outre un nouvel argument, d'ordre chronologique, fort convaincant : aucun des documents mentionnant l'existence d'un koinon du Pont n'est antérieur au règne de Trajan et donc l'hypothèse selon laquelle un koinon du Pont occidental aurait précédé un koinon du Pont oriental ne tient plus. On peut également mentionner le fait que les deux cités (Heraclea Pontica ou Amastris) qui sont supposées avoir pu être la capitale d'un koinon du Pont occidental ne font quasiment pas référence - dans leurs émissions monétaires - au fait qu'elles auraient pu occuper ce rang. En effet, à la différence de Neocaesarea,Amastris n'a pas le titre de néocore et n'a frappé aucun type à connotation agonistique. En ce qui concerne Heraclea Pontica, le titre de néocore n'apparaît qu'à partir de Philippe ${ }^{17}$, et des types avec des

15. Ces représentations avec six Tychès appartiennent aux règnes de Septime Sévère et de Géta. Contrairement à ce qu'écrit X. Loriot (2006, p. 529, n. 2) ce type n'est pas si rare que cela, puisqu'il n'y a pas un, mais quatre coins de revers différents (et non cinq comme le prétend Z. Çizmeli, les revers des monnaies nos 51 et 52 étant en fait les mêmes). Voir Çızmeli 2006, nos $51,52,53,215$ et 216 .

16. DALAison 2008, p. 22.

17. Waddington, Babelon, Reinach 1908, p. 380, no 231 sqq. 
urnes de jeux ne sont présents que sous Gallien ${ }^{18}$. Il semblerait donc qu'il faille retenir comme hautement plausible l'idée d'un unique koinon du Pont créé fin Ier-début IIe siècle apr. J.-C. et dont la capitale serait Neocaesarea. Il comprendrait les cités d'Heraclea Pontica, Tieion, Amastris, Abonoteichos-Ionopolis, Sinope, Amisos, Polemonium, Cerazus, Trapezus, Neocaesarea, Amaseia, Comana Pontica, Zela, Sebastopolis et Sebasteia.

Néanmoins, nous ne constatons aucune unité dans les types monétaires des cités du Pont Galatique et du Pont Polémoniaque, même si la Tychè se retrouve fort logiquement sur le monnayage d'Amaseia, Neocaesarea, Trapezus et Zela et si Athéna et Zeus Nicéphore apparaissent à Amaseia et Zela. Sur leurs monnaies, Comana Pontica et Sebastopolis sont quasiment «spécialisées » et honorent essentiellement une divinité ${ }^{19}$ : Mâ, à Comana Pontica, Héraclès, à Sebastopolis. Les types monétaires des quatre autres cités pontiques sont relativement plus varié $^{20}$ : Apollon, Asclépios, Athéna, Hadès-Sarapis, Héra (?), Nikè, Tychè, Zeus Nicéphore, Zeus Stratios sous la forme d'un bûcher ${ }^{21}$ (variantes), aigle, fleuve, serpent, temple(s), à Amaseia ; Mèn, Tychè, Démos, temple(s), à Neocaesarea ; Dionysos, Mithra (variantes), Sérapis, Tychè, à Trapezus ; Anahita-Anaiitis, Athéna, Hélios, Tychè, Zeus Epikarpios, Zeus Nicéphore, autel, temple(s), tours, à Zela.

Sans doute fières de la beauté de leurs monuments religieux, les autorités municipales d'Amaseia, Comana Pontica, Neocaesarea, Sebastopolis et Zela les ont fait représenter sur leurs monnaies. La présence d'une divinité sur les revers monétaires d'une cité n'indique pas nécessairement qu'elle y possédait un temple. La liste des édifices religieux n'est donc pas forcément la même que celle des dieux et déesses représentés sur les pièces ${ }^{22}$. En revanche, lorsque des coins de revers montrent des divinités placées sous des éléments architecturaux, nous pouvons supposer que ces dernières avaient un sanctuaire dans la ville. Toutefois, comme le notait déjà Julie Dalaison ${ }^{23}$, «il paraît hasardeux de s'appuyer sur les seules monnaies pour restituer les superstructures antiques ${ }^{24}$. Dans les cinq cités sont attestés les monuments suivants :

- à Amaseia ${ }^{25}$, trois divinités sont associées à des édifices : sous la forme du serpent, Asclépios est placé sur un autel situé au centre d'un temple distyle à fronton trapézoïdal sur des degrés (R 328) - accompagné de Cerbère,

18. Waddington, Babelon, Reinach 1908, p. 382, nos 245-246.

19. On retrouve aussi un aigle de face à Comana Pontica et la Tychè à Sebastopolis.

20. Nous n'avons pas pris en compte dans cette recherche les types monétaires se rapportant de près ou de loin au seul culte impérial.

21. Voir Dalaison 2010, p. 203-218.

22. Sur la représentation des monuments religieux sur les monnaies, et leur valeur informative ou symbolique, voir HARL 1987 et BelayCHE 2003, p. 111-138.

23. DaLAison 2008, p. 174.

24. Sous Domitien et Sévère Alexandre, le même temple placé au centre de la ville est soit tétrastyle (R 15-18; R 451, 453), soit pentastyle (R 452), soit hexastyle (R 451, 453).

25. Voir Dalaison 2008, p. 171-179. 
Hadès-Sarapis est assis sur un trône à dossier placé sous une arcade tétrastyle $(\mathrm{R} 234)^{26}$ - Tychè est debout sur une base à l'intérieur d'un temple distyle (R 282) et sous une arcade tétrastyle surmontée d'un fronton ( $R$ 442), mais il s'agit probablement du même temple. Sur des revers de Trajan (R 23-30), on trouve des temples tétrastyles, de face sur des degrés, avec colonnes cannelées ou non, qui sont difficilement identifiables (culte impérial municipal ?).

- à Comana Pontica ${ }^{27}$, seule Mâ est associée à un sanctuaire ; Mâ dans un temple tétrastyle $(\mathrm{R} 15,16)$ - temple tétrastyle à fronton échancré avec bétyle de Mâ surmonté d'une Victoire (R 19) - temple tétrastyle avec bétyle de Mâ surmonté d'une Victoire (R 23-40) - temple tétrastyle (R 41). Il est probable qu'il n'y avait dans la ville qu'un seul sanctuaire de Mâ.

- à Neocaesarea ${ }^{28}$, sont représentés un temple tétrastyle (R 1-25...) avec différentes variantes et un temple pentastyle (R $41,42,68 \ldots$.. Il s'agit probablement du même sanctuaire du culte impérial provincial ${ }^{29}$.

- à Sebastopolis ${ }^{30}$, seul Héraclès est associé à un temple tétrastyle sur un podium ; à l'intérieur, Héraclès avec la massue et la peau de lion (R 5, 24, 28 ...) Héraclès avec la massue et la peau de lion sous une arcade qui relie deux temples tétrastyles (R 7,8...)-temple tétrastyle (R 16-18). Ce (ou ces) temple(s) tétrastyle(s) devai(en)t être consacré(s) à Héraclès, la divinité éponyme de la cité qui prit, à partir d'Hadrien ${ }^{31}$, le nom double de Sebastopolis-Heracleopolis pour honorer Héraclès son fondateur mythique.

- à Zela ${ }^{32}$, seule la Tychè est associée à un monument : temple distyle avec fronton triangulaire ; au centre, autel allumé (R 96) - temple tétrastyle avec arcade centrale et fronton triangulaire ; au centre, autel allumé $(\mathrm{R} 9,28,58)$ ou inscription (R 10) ou guirlande (R 61)... - temple tétrastyle avec fronton triangulaire sur un rocher ( $\mathrm{R} 83)$ - temple tétrastyle avec arcade centrale et fronton triangulaire ; au centre, Tychè (R 29) - temple hexastyle à fronton échancré au milieu (R 11-16...) - deux tours crénelées reliées par un temple distyle avec fronton triangulaire sur un rocher ( $\mathrm{R} 82)$. Le temple tétrastyle avec la statue de Tychè lui était clairement consacré. Qu'en allait-il du (ou des) autre(s) édifice(s) ? Il faut sans doute penser qu'il y avait au moins parmi eux un sanctuaire d'Anahita-Anaïtis.

26. La présence d'un temple de Sérapis à Amaseia est confirmée par la mention d'un Serapeion dans la vie de saint Basilicus (Acta Sanctorum 1668, p. 238).

27. Voir AMANDRY, RÉmy 1999, p. 51-53.

28. Çizmeli 2006, p. 118-123.

29. Sous Gordien III (R 24 et 25) et Tranquilline (R 13), Mèn serait représenté dans un temple tétrastyle. Il est plausible d'envisager que le culte impérial ait été installé dans le temple du dieu local (ÇIZMELI 2006, p. 123).

30. AMANDRY, RÉmy 1998, p. 38-42.

31. AmANDRY, RÉmy 1998, p. 7-8.

32. Dalaison, RÉmy, AmandRy 2009, p. 37-41. 
Dans le panthéon «monétaire» des différentes cités pontiques, nous retrouvons des divinités d'origine indigène (Mâ, Mèn, Zeus Stratios), des divinités d'origine iranienne (Anahita-Anaïtis, Mithra) et des divinités apparemment gréco-romaines (Apollon, Asclépios, Athéna, Nikè, Tychè...). Il vaut la peine de remarquer que, sauf Sebastopolis, les cinq autres cités pontiques honorent sur leur monnayage une divinité d'origine indigène ou iranienne. Il a donc semblé intéressant de regarder de plus près cet aspect particulier.

\section{Le culte d'Anahita-Anaiitis à Zela}

La déesse Anahita-Anaïtis n'apparaît formellement que sur deux types de revers dans tout le monnayage de Zela : sous Trajan, elle est assise à gauche, drapée, la tête voilée, coiffée d'un croissant et tenant une gerbe d'épis dans la main droite $^{33}$; sous Caracalla, elle est debout de face, drapée, la tête voilée, tenant un épi dans la main droite et un objet indistinct dans la main gauche ${ }^{34}$. En dehors de ces quelques monnaies, on est en droit de penser que les très nombreux revers représentant un temple hexastyle à fronton échancré renvoient au sanctuaire d'Anahita-Anaïtis. Sur des pièces de module plus petit, il est également possible que le temple ${ }^{35}$ distyle ou tétrastyle ${ }^{36}$, parfois placé sur un rocher, fasse aussi référence à ce sanctuaire. En effet, la place réduite offerte par le flan monétaire ne permettait pas de représenter un temple hexastyle et la volonté de faire figurer un autel dans le temple ou de placer le sanctuaire entre deux tours justifiait sans doute la réduction du nombre de colonnes en façade. Dans la réalité, il n'est pas du tout sûr que le temple d'Anahita-Anaïtis ait été hexastyle, car les monnaies font la part belle à une certaine «licence artistique» et l'essentiel était en fait que le type soit immédiatement reconnaissable par les utilisateurs des pièces ${ }^{37}$.

L'importance du culte d'Anahita-Anaïtis à Zela est confirmée par deux passages de $\operatorname{Strabon}^{38}$. Dans le premier l'auteur évoque les Saces qui se seraient avancés jusqu'en Cappadoce pontique. Ils auraient été anéantis par les Perses, qui auraient alors, sur le lieu de leur défaite, entassé de la terre autour d'un rocher qui se trouvait dans la plaine pour former une colline artificielle. Ils y auraient dressé des

33. Dalaison, Rémy, Amandry 2009, nos 1 et 2. La déesse est formellement identifiée par la légende de revers.

34 . Voir appendice et planche, figure 2 .

35. Sous Caracalla : Dalaison, Rémy, Amandry 2009, nos 106 et 107 ; sous Géta : Dalaison, RÉMY, AMANDRY 2009, no 137.

36. On ne sait pas exactement à quel sanctuaire renvoyaient les autres temples tétrastyles figurés sur les monnaies de plus grand module. Comme dit plus haut, quand Tychè est représentée dans le temple, il est fort probable que celui-ci lui était dédié. Quand le temple ne comporte en son centre qu'un autel, ou un treillis, ou une guirlande, il est difficile de savoir à quelle divinité il était consacré.

37. Voir n. 22.

38. Strabon, Géographie, 11, 8, 4-5 et 12, 3, 37. 
remparts et un sanctuaire dédié à Anaïtis, Omanos et Anadatos - cette assertion est un argument pour identifier le temple tétrastyle ou distyle placé sur un rocher, et évoqué plus haut à propos des monnaies de Caracalla, avec le sanctuaire d'Anahita-Anaïtis. Les Perses instaurèrent également la fête annuelle des Sacées, toujours célébrée par les habitants de Zela. Selon Strabon, il existe également une seconde version : Cyrus aurait déclaré la guerre aux Saces et aurait été battu. Il serait alors reparti vers son camp situé dans la plaine de la future Zela, avant de quitter cette zone et de laisser son campement vide. Les Saces seraient alors arrivés et se seraient enivrés dans le camp. Revenu sur ses pas, Cyrus les aurait anéantis. Attribuant aux dieux cette victoire, le roi perse aurait consacré ce jour à la déesse tutélaire de sa dynastie sous le nom de fête des Saces. Le sanctuaire de Zela est décrit comme une principauté sacerdotale avec ses esclaves sacrés ${ }^{39}$. La ville devient une polis sous Pompée qui la dote d'un territoire. Dans son second passage sur Zela, Strabon écrit que la butte artificielle sur laquelle est placée Zela est due à la reine Sémiramis, qu'Anaïtis est également adorée par les Arméniens et que c'est à Zela que les habitants du Pont prononcent leurs serments les plus importants. Il revient ensuite sur l'organisation de la principauté sacerdotale ${ }^{40}$ et sur l'évolution de son territoire et de son statut, de Pompée à Pythodoris.

Venu de Perse et sans doute passé par l'Arménie, le culte d'Anahita, aurait donc été introduit en Asie Mineure, soit au vie siècle av. J.-C. par Cyrus ${ }^{41}$, soit un peu plus tard au Ive siècle av. J.-C. par Artaxerxès II ${ }^{42}$, connu pour avoir favorisé son culte dans toutes les capitales de l'empire perse. Il aurait connu un grand succès en Anatolie, grâce, en particulier, à la présence de colonies perses, ayant survécu à la conquête macédonienne. Déesse de l'eau comme élément fécondant, de la prospérité - symbolisée par l'épi de blé sur la monnaie de Caracalla à Zela - et de la procréation, Anahita a vu son nom «grécisé » sous la forme d'Anaïtis ${ }^{43}$, et a été assimilée à certaines déesses-mères, appelées Meter ou Artémis. Pour autant, la divinité continue à porter son nom - ou sa déformation grecque - et ne perd pas ses caractéristiques originelles ${ }^{44}$. Dans le sanctuaire de Zela, elle est donc associée à deux «démons » d'origine iranienne, Omanos et Anadatos. Si le premier peut être identifié au perse Vohumano - esprit protecteur des animaux -, on ne sait

39. Voir Dignas 2002, Bernadet 2009, p. 63-87 et Bernadet 2011, p. 293-317.

40. Voir Debord (1982, p. 55-59, 122, 164 et 173) et SöKmEn (2009, p. 277-287) pour le fonctionnement précis de la principauté sacerdotale de Zela.

41. BRIANT 1996, p. 264-265 ; SöKMEN 2009, p. 281.

42. Debord 1982, p. 265-267. Si elle date l'introduction du culte d'Anahita en Asie Mineure du $\mathrm{VI}^{\mathrm{e}}$ siècle, E. Sökmen $(2009$, p. 281) pense en revanche que le temple de Zela a été bâti à la période tardo-achéménide, soit au $\mathrm{IV}^{\mathrm{e}}$ siècle.

43. Mitchell 2007, p. 169.

44. TEIXIDOR 1981,p. 754-756 ; DEBORD 1982,p. 265-267. R. Turcan (1992,p. 197 et 253) précise que la déesse a aussi été identifiée à la Lune. Cela est peut-être à mettre en relation avec le fait qu'en Mésopotamie Anahita a été assimilée à Ištar (BRIANT 1996, p. 264-265 ; MAREK 2010, p. 335336). Ce lien avec la Lune est en tout cas bien visible sur les monnaies de Trajan émises à Zela. 
rien du second ${ }^{45}$. À l'époque romaine, le culte d'Anahita-Anaïtis reste encore bien vivant à Zela, où son sanctuaire est un des plus importants d'Asie Mineure. Il est illustré sur une large majorité des types monétaires émis par la cité qui y voit un moyen de mettre en avant sa spécificité.

\section{Le culte de Mâ à Comana Pontica}

Les revers des monnaies de Comana du Pont frappées sous les premiers Sévères sont les seules représentations connues de Mân ${ }^{46}$. La déesse apparaît sous deux aspects principaux. Elle peut être présentée de manière anthropomorphe : elle est alors debout de face, la tête radiée, tenant un bouclier et une massue ${ }^{47}$; la divinité est le plus souvent dans un temple, mais cette figuration n'est pas systématique. Elle peut également être évoquée par son bétyle surmonté d'une Victoire et placé dans un temple. Dans un cas $^{48}$, le bétyle cylindrique de Mâ est entouré de guirlandes portées par des figures féminines ; il est surmonté d'un dôme flanqué de deux anneaux de portage et lui-même surmonté d'une Victoire. On peut ainsi supposer que le temple de Mâ à Comana abritait à la fois une statue de la divinité et son bétyle. C'est sans doute ce dernier qui était porté dans une sorte de châsse cylindrique et qui était exposé aux yeux de tous lors des exodes de la déesse dans la cité.

Attesté uniquement dans les deux Comana d'Anatolie - Comana de Cappadoce et Comana du Pont -, le culte de Mâ est décrit par Strabon dans sa Géographie ${ }^{49}$. Il écrit qu'Enyo et Mâ seraient une même divinité et attribue l'introduction du culte, dont les rites seraient identiques à ceux d'Artémis Tauropole, à Oreste et Iphigénie qui l'auraient ramené de Scythie Taurique. Le nom des cités proviendrait du don par Oreste de sa chevelure (komè), «qu'il avait laissé croître en signe de deuil». Ces récits étiologiques sont évidemment à prendre avec une grande précaution. Dans sa description de Comana du Pont, Strabon est un peu plus prolixe, puisqu'il évoque de façon assez précise le fonctionnement de l'état-temple, les prostituées sacrées, les fêtes et les deux exodes annuels de la déesse.

Jusqu'à l'époque romaine, les deux Comana, étaient organisées en principautés sacerdotales ${ }^{50}$. Sous Mithridate Eupator, à Comana du Pont ${ }^{51}$, le prêtre occupait la seconde place après le roi. À partir de Pompée, Comana est toujours dirigée

45. Olshausen 1990, p. 1870-1871.

46. Voir appendice et planche, figures 3-6.

47. Il se pourrait aussi que la déesse porte une massue et un arc (AMANDRY, RÉmy 1999, monnaie no 60), mais l'identification du type est incertaine.

48. AmANDRY, RÉmy 1999, monnaie no 21.

49. Strabon, Géographie, 12, 2, 3 (pour Comana de Cappadoce) et 12,3,32-36 (pour Comana du Pont).

50. Voir en particulier Debord (1982, p. 55-59, 122 et 234) et SöKmen (2009, p. 277-287) pour de plus amples détails sur l'organisation des principautés sacerdotales.

51. AMANDRY, RÉmY 1999, p. 14-15. 
par son grand prêtre - désormais nommé par les autorités romaines -, mais elle est devenue une principauté-cliente de Rome, dont le territoire et la population - qui n'était pas uniquement composée de hiérodules - ont été augmentés. Après son intégration dans l'empire romain, en 34 p.C., Comana devient une polis dans laquelle le grand prêtre continue sans doute à jouer un rôle important.

Malgré ce qu'en dit Strabon, il semblerait que Mâ soit à l'origine une divinité hittite $^{52}$. Elle a essentiellement des caractères guerriers (massue, bouclier) et solaires (couronne radiée). Son aspect belliqueux est confirmé par son identification avec Enyo et Bellone ${ }^{53}$ et il semblerait que, avec le temps, cette dernière fonction l'ait emporté sur la fonction solaire. Le culte de Mâ aurait été introduit à Rome après la guerre de Sylla contre Mithridate Eupator et aurait été assimilé à celui de Bellone et de Virtus.

Mâ a donc un caractère hybride. Dans sa région d'origine, l'est de l'Anatolie, elle garde sa dénomination indigène, mais elle a subi des influences grecques et romaines, visibles - sur les monnaies - dans la représentation anthropomorphe de la déesse. Cet aspect est d'ailleurs assez tardif, puisqu'il n'est connu que pour la période romaine. Toutefois, ses spécificités originelles sont toujours bien attestées, en particulier par la présence du bétyle. Mâ est donc loin d'avoir été totalement assimilée à une déesse gréco-romaine. Son culte avec ses caractéristiques propres est toujours bien vivace à Comana du Pont, puisqu'il est le thème quasi exclusif du monnayage émis par la cité ${ }^{54}$. Ces monnaies étaient d'ailleurs sans doute en partie destinées aux pèlerins et aux visiteurs du temple lors des grandes fêtes de la déesse ${ }^{55}$. On faisait ainsi figurer sur les monnaies la raison même de l'existence de la cité et le symbole de son identité et de sa prospérité.

\section{Le culte de Mèn à Neocaesarea}

Pendant toute la période de production de l'atelier de Neocaesarea, Mèn n'apparait que sur un seul coin de revers de Géta en 205-206 p.C. ${ }^{56}$ Le dieu a un croissant de lune sur les épaules, un sceptre à la main et il porte un chiton, un pantalon et un bonnet phrygiens ${ }^{57}$. Il est debout devant le couple formé par l'empereur - cuirassé avec un sceptre à la main - couronnant la Tychè de la cité ${ }^{58}$.

52. Olshausen 1990, p. 1886-1887 ; Proeva 1992, p. 330-331.

53. Faite par J. Teixidor (1981, p. 754), l'identification entre Mâ, Cybèle et Anahita est réfutée par N. Proeva (1992, p. 330-331).

54. AMANDRY, RÉMY 1999, p. 19.

55. Cela ne veut pas dire pour autant que les monnaies avaient uniquement une fonction commémorative. Elles n'étaient pas simplement des médailles ; elles avaient aussi un rôle économique évidemment compatible avec l'expression de la fierté civique sur les revers monétaires.

56. Il se peut également qu'il apparaisse placé dans un temple sur des monnaies de Gordien III et de Tranquilline. Voir ici n. 29.

57. VollKommer 1992a, p. 462-472.

58. Voir appendice et planche, figure 7. 
Mèn n'est qu'un «simple» spectateur de la scène, qui indique que l'empereur est la source de légitimité de la cité à laquelle, par une sorte de lien privilégié, il apporte la paix et la prospérité. Cette présence très ténue et discrète du dieu dans le monnayage est sans doute à mettre en relation avec la place éminente qu'occupe dans le Pont, à partir de Trajan, la cité de Neocaesarea en tant que capitale du koinon. Avant les émissions des premiers Sévères, la production de l'atelier monétaire a été assez faible et sporadique et Mèn n'apparaît jamais ; ensuite, les types monétaires sont quasiment consacrés exclusivement au koinon et aux néocories de Neocaesarea. Le rang occupé par la cité et son exaltation iconographique ont pris le pas sur les autres représentations et constituent désormais la «carte de visite» de la ville.

Cependant, la présence de Mèn à Neocaesarea n'est pas totalement fortuite et anecdotique. En effet, Strabon ${ }^{59}$ - le témoignage littéraire essentiel sur le culte du dieu -, quand il parle de Cabeira (devenue cité sous le nom de Diospolis avec Pompée, puis de Sebastè avec Pythodoris et enfin de Neocaesarea au moment de son incorporation dans l'empire romain ${ }^{60}$ ), évoque la présence d'un sanctuaire de Mèn Pharnakou (associé à Sélénè) sur le territoire de la cité, dans le bourg urbain (komopolis) d'Ameria. Cette agglomération abritait une principauté sacerdotale ${ }^{61}$, organisée sur le modèle de celles de Zela ou de Comana Pontica. De plus, Mèn jouait un rôle important dans le royaume du Pont, puisque le serment royal était prêté au nom de la Tychè du roi et de Mèn Pharnakou. Il était donc le dieu de la justice entre les hommes, mais aussi, par le symbole de la lune qui croit et qui décroit, un dieu de la renaissance et de la fertilité62, dont l'action était sans doute renforcée par la présence de sa parèdre Sélénè.

Les origines du dieu Mèn font l'objet de nombreuses discussions et il ne paraît pas réellement possible de trancher. La synthèse la plus récente sur le sujet est celle de Guy Labarre ${ }^{63}$. Prenant le contrepied des théories avancées en particulier par E. N. Lane ${ }^{64}$ d'une origine indo-persique de Mèn, l'auteur réfute cette idée, car Mèn n'est pas forcément identique au dieu perse Mao ${ }^{65}$. Quoi qu'il en soit, deux points sont indiscutables : d'une part, le succès du culte de Mèn en Phrygie et dans la colonie romaine d'Antioche de Pisidie, où a été retrouvée une importante documentation (inscriptions et reliefs) ${ }^{66}$; d'autre part, le silence des sources sur Mèn jusqu'à l'arrivée des Grecs. La représentation anthropomorphique du dieu daterait de la période grecque. Pour Guy Labarre, le culte de Mèn était anatolien ; son succès est «le résultat de la rencontre d'une croyance indigène avec les Grecs

59. Strabon, Géographie, 12, 3, 31.

60. ÇizMELi 2006, p. 84-85.

61. Debord 1982, p. 122 et 164 ; SöKMEN 2009, p. 277-287.

62. TurCAN 1992, p. 69 ; LABARRE 2010, p. 34-36 et 45-50.

63. LABARRe 2009 et 2010. Voir aussi Mitchell 2007a, p. 161-162.

64. LANE 1967-1968, p. 81-98.

65. LABARRE 2010, p. 61-69.

66. Byrne, LabarRe 2006 ; Labarre 2010, p. 27-34. 
qui lui ont apporté les moyens de se révéler ${ }^{67}$. Il est plausible de penser que le culte a été exporté dans le Pont par l'intermédiaire de marchands ou d'esclaves ${ }^{68}$.

La présence de l'épiclèse Pharnakou (terme d'origine perse) fait sans doute référence au nom du fondateur du sanctuaire. Il peut s'agir, soit de Pharnace, mari d'Atossa, tante de Cyrus, et fondateur légendaire des dynasties pontique et cappadocienne, soit de Pharnace I ${ }^{\mathrm{er}}$, roi du Pont entre 189/188 et 169 a.C.

Les origines et l'évolution du culte dans le Pont dépendent évidemment de l'hypothèse retenue ${ }^{69}$. En faveur de la seconde plaide peut-être le fait qu'une monnaie de la ville de Pharnakeia dans le Pont (fondation de Pharnace ${ }^{\mathrm{er}}$ ) représente au droit Mèn ${ }^{70}$. Cette monnaie daterait des années 150-125 a.C. et serait l'expression du lien entre Pharnace $\mathrm{I}^{\mathrm{er}}$ et Mèn Pharnakou. Cependant, on pourrait aussi supposer que les autorités aient voulu profiter du fait que la divinité portait l'épithète Pharnakou (qui aurait renvoyé à une origine plus ancienne) pour affirmer un lien entre Pharnace $\mathrm{I}^{\mathrm{er}}$ et le dieu. On a d'ailleurs parfois voulu voir dans la représentation de symboles lunaires et peut-être solaires ${ }^{71}$ (croissant de lune surmonté d'une étoile) sur un certain nombre de monnaies de la dynastie des Mithridatides une illustration de cette relation entre certains dieux locaux, dont Mèn, et la dynastie des Mithridatides ${ }^{72}$. Pour Eckart Olshausen, la lune renverrait à Mèn et l'étoile à Zeus Stratios. Il y aurait alors une sorte d'amalgame qui ferait écho aux composantes et aux fondements religieux de la royauté. Le croissant et l'étoile deviendraient des «armoiries » de la dynastie, voire du territoire pontique. S'il est sûr que ces deux symboles ont une signification importante à l'époque des Mithridatides et s'il est certain que l'aspect iranien et la théologie astrale ont joué un rôle notable, il est en revanche hasardeux d'établir une connexion directe et unique entre symboles et divinités. Quoiqu'il en soit, la forte présence de Mèn dans le panthéon du royaume du Pont ne semble pas avoir perduré pendant la période romaine ; le dieu a été supplanté, dans la cité où se trouvait son sanctuaire, par l'importance du koinon et du culte impérial provincial, qui occupait désormais la première place ${ }^{73}$. La frappe de Géta pourrait être un simple rappel historique.

67. LABARRE 2010, p. 69.

68. LABARRE 2010, p. 142-143.

69. LABARRE 2010, p. 36-37 et 64.

70. Waddington, Babelon, Reinach 1925,p. 138, no 1. Voir aussi OlshausEn 1990, p. 1887-1889 et Michels 2009, p. 193-196 (ce dernier interprète également les revers des monnaies de Pharnace comme des représentations de Mèn Pharnakou, mais cela reste très hypothétique. Voir ici n. 88).

71. L'étoile peut être interprétée soit comme un symbole lunaire, soit comme un symbole solaire (car il ne s'agirait plus alors d'une étoile à proprement parler, mais de la représentation du soleil, qui est d'ailleurs une étoile).

72. Olshausen 1990, p. 1887-1889; Michels 2009, p. 186-190.

73. Il est d'ailleurs possible que le culte impérial et le culte de Mèn aient cohabité dans le même temple. Voir Çızmeli (2006, p. 122-124) qui évoque également la mention du sanctuaire de Mèn Pharnakou par Grégoire le Thaumaturge, ce qui prouverait que le culte, même s'il a perdu de sa vitalité, était toujours présent dans la cité au $\mathrm{III}^{\mathrm{e}}$ siècle apr. J.-C. 


\section{Le culte de Mithra à Trapezus}

Si les textes littéraires sur Mithra ne mentionnent jamais Trapezus, les représentations du dieu sont en revanche très fréquentes sur le monnayage de la cité, aussi bien sous les premiers Sévères ${ }^{74}$ que pendant les autres périodes d'activité de l'atelier. Mithra peut être figuré en buste drapé à droite, coiffé du bonnet phrygien radié, avec, devant lui, le protomé de son cheval, mais le plus souvent, il est à cheval à droite avec un autel devant lui. Peuvent également s'ajouter un arbre, une colonne au sommet de laquelle se trouve un oiseau - sans doute le corbeau, messager du soleil, qui a prescrit l'immolation du taureau -, et parfois les dadophores, Cautès et Cautopatès, abaissant et levant respectivement leur torche. À la différence d'autres sites du monde romain où le culte de Mithra est connu, on ne trouve jamais à Trapezus de représentation de Mithra en divinité tauroctone. Cette imagerie de Mithra à cheval est une spécificité de Trapezus ; elle n'est jamais attestée ailleurs dans le «mithriacisme romain », ce qui rend difficile son interprétation ${ }^{75}$. Elle peut être rapprochée de certaines représentations de Doura Europos ou de Rhénanie, mais là il s'agit plutôt de scènes de chasse ${ }^{76}$. Il semble donc préférable de retenir l'hypothèse, suggérée par Nicole Belayche, d'une contamination, au moins iconographique, des représentations anatoliennes (ou thraco-anatoliennes) de dieux masculins cavaliers ${ }^{77}$.

Pour autant les différents éléments apparaissant sur les revers des monnaies de Trapezus n'ont rien d'exceptionnel et sont tout à fait caractéristiques du culte du Mithra gréco-romain ${ }^{78}$. Les Cautès et Cautopatès, symbolisent le soleil levant et le soleil couchant. Le corbeau est le messager du soleil, qui a prescrit l'immolation du taureau. Les arbres (ainsi que les autres plantes et animaux) renvoient au monde végétal et animal. Ces modes de représentation ne viennent pas de l'art perse, mais sont des réinterprétations, voire des ajouts, de l'époque grecque et romaine. Vieille divinité perse, connue des Grecs dès le ve siècle av. J.-C., Mithra n'a jamais connu un grand succès en Asie Mineure ${ }^{79}$, même si quelques découvertes ont été faites (par exemple à Pergé en Pamphylie). Dès l'époque hellénistique, l'imagerie, l'idéologie et la «geste» de Mithra - qui est en fait une histoire du monde et de la création ${ }^{80}$ - ont connu une très sensible évolution. Il est devenu

74. Voir appendice et planche, figures 8-9.

75. VOLLKOMMER 1992b, p. 583-626.

76. TURCAN 1992, p. 245-246 ; TURCAN 1993, p. 61 ; WoJAn 2006, p. 190-191.

77. De même, Mèn est souvent représenté à cheval sur les monnaies. Voir VolLKOMMER 1992a, p. 468-469 et LABARRE 2010, p. 31-32.

78. TURCAN 1992, p. 193-241; TuRCAN 1993.

79. Le voyage d'exploration de Franz et Eugène Cumont dans le Pont est une illustration de cette faible diffusion du mithriacisme en Anatolie. En effet, Nicole Belayche (2012, p. XLVII et LXV-LXVI), quand elle évoque ce séjour des deux frères, écrit que «la moisson mithriaque fut très modeste».

80. Turcan 1992, p. 217-227 ; TurCan 1993, p. 45-72 et 93-114; Beard, North, Price 2006, p. 268-278. 
un culte initiatique à mystères en lien avec l'entrée et la sortie de l'âme du monde des vivants. Comme le notait déjà Robert Turcan ${ }^{81}$, «si Mithra est iranien, le mithriacisme est gréco-romain ».

Les origines du dieu et sa forme primitive sont plus difficiles à appréhender. En Perse, Mithra était un dieu du serment, du contrat, un dieu guerrier, protecteur des campagnes et des récoltes. C'était une divinité de la prospérité et de la lumière ${ }^{82}$. Mais, chez les Perses, il n'est pas du tout certain que Mithra ait été assimilé au Soleil et il se peut que cette association ait été créée a posteriori, lorsqu'à la période romaine, Mithra a été identifié à Sol Invictus. Le lien entre Mithra et la lumière a parfois fait écrire à certains ${ }^{83}$ que Mithra pouvait être identifié à Ahura Mazda (lui aussi associé à la lumière). Cette tendance syncrétique qui vise à faire de Mithra, Ahura Mazda et Hélios une seule et même divinité est très certainement à rejeter, dans la mesure où il est évident que certains attributs peuvent être communs à différentes divinités ${ }^{84}$.

La présence et l'origine du culte de Mithra dans le Pont ont donné lieu à de nombreuses hypothèses. Il faut d'abord noter que, quelle que soit la période considérée, les traces de l'existence du culte de Mithra dans cette région sont relativement ténues, si ce n'est dans le nom de six souverains du royaume qui s'appellent Mithridate, littéralement «donné par Mithra». Selon Stephen Mitchell ${ }^{85}$, le culte de Mithra aurait été introduit en Asie Mineure par les Iraniens, mais il n'existe pas de preuve formelle pour étayer cette hypothèse. Robert Turcan suppose que c'est peut-être dans le Pont, vers les années 100-80 a.C.,que se serait affirmée l'imagerie gréco-romaine de Mithra, celle d'un dieu vêtu à l'orientale et immolant le taureau ${ }^{86}$. Eckart Olshausen ${ }^{87}$ quant à lui se demande si le culte de Mithra n'aurait pas fait son apparition dans le Pont seulement à l'époque romaine, car, avant la frappe des monnaies provinciales romaines de Trapezus, il n'y a pas de trace réelle du culte dans la région. À part le nom des rois, il n'y a rien de concret, sauf si l'on veut reconnaître - ce qui est loin d'être assuré - Mithra dans la figure masculine qui figure sur des monnaies de Pharnace $\mathrm{I}^{\text {er }}{ }^{88}$. Toutefois, le dieu apparaît

81. TURCAN 1993, p. 95.

82. TURCAN 1992, p. 193-194 ; BRIANT 1996, p. 262-264.

83. TurCan 1992, p. 194-197. Voir ce qu'en pense Briant 1996, p. 263.

84. C'était l'hypothèse de Fr. Cumont (1901, p. 47-57 et 1906, p. 171-184). Voir maintenant BECK 2004 et 2006

85. Mitchell 1993, p. 29-30. C'est une simple reprise de l'hypothèse de Fr. Cumont pour laquelle il n'y a aucune preuve.

86. TurCAN 1993, p. 50. Cette hypothèse est cependant sujette à caution, dans la mesure où, dans le Pont et à Trapezus, Mithra tauroctone n'est jamais représenté.

87. Olshausen 1990, p. 1889-1890.

88. Waddington, Babelon et Reinach 1925, p. 11-12, nos 4-5. Chr. Michels (2009, p. 193) écrit que l'indentification du personnage avec Mithra est discutée et qu'il est parfois reconnu en tant qu'Aion, Mâ, Hermès-Mithra, voire Hermès-Mithra-Dionysos ou Mèn Pharnakou - hypothèse qui remporte les suffrages de l'auteur (voir ici n. 70). 
de façon certaine sur des pièces de Zela ${ }^{89}$, datées de la fin des guerres mithridatiques. Cependant, il n'est pas possible de savoir à quand exactement remonte l'instauration du culte de Mithra dans le Pont.

D'origine iranienne, ce dieu s'est fortement teinté de culture gréco-romaine et est en tout cas bien représenté dans la cité de Trapezus qui jouait un rôle important dans le dispositif militaire de l'Empire à l'époque romaine. Port de commerce, la ville était aussi un port militaire pour le transit de troupes vers le limes de l'Euphrate. C'était peut-être aussi la base navale de la classis Pontica, où tout au moins un de ses ports relais ${ }^{90}$. Toutefois, comme Nicole Belayche nous l'a fait remarquer, «si Mithra était passé par les soldats, il serait tauroctone et on aurait des dédicaces, puisque tel était leur mode d'expression. Or il est à cheval, donc d'iconographie "anatolisante", et nous n'avons que les monnaies».

\section{Le culte de Zeus Stratios à Amaseia}

Nous ne reprendrons pas ici en détail ce qui a déjà été écrit ailleurs par Julie Dalaison $^{91}$. Nous nous contenterons de rappeler les principales caractéristiques de ce dieu et d'apporter quelques éléments nouveaux. La présence du culte de Zeus Stratios dans la cité d'Amaseia est affirmée par l'épigraphie, la numismatique et les vestiges archéologiques du site de Yassiçal.

Sur les revers des monnaies, Zeus Stratios est toujours évoqué par un bûcher et jamais par une représentation anthropomorphe ${ }^{92}$. Parmi toutes les divinités étudiées dans cet article, Zeus Stratios est ainsi la seule pour laquelle on n'a pas cherché à représenter physiquement le dieu - si ce n'est par ses symboles -, mais son culte. Le bûcher est donc central, car il incarne le culte civique. Il est l'expression de l'identité et de la vie politique à travers les cérémonies qui se déroulent à Yassiçal où l'on a retrouvé un mur d'enceinte entourant une plateforme sur laquelle se dressait le bûcher qui était reconstruit pour chaque cérémonie. Ce sanctuaire servait de lieu de rassemblement pour tous les habitants de la cité et c'est au moment de l'embrasement du bûcher que se manifestait l'activité civique. Les rituels en l'honneur de Zeus Stratios sur un bûcher en plein air sont évoqués par Appien pour l'époque de Mithridate VI Eupator ${ }^{93}$ et ne sont pas sans rappeler les mœurs religieuses perses telles qu'elles sont décrites par Strabon ${ }^{94}$.

Ce bûcher peut être à un ou deux étages ; il est toujours accompagné d'un arbre et divers éléments peuvent être placés sur ou à proximité du bûcher - quand il est à un étage - : un aigle éployé ; un bœuf couché sur le dos ; un quadrige de face, dans lequel se trouve en général un personnage tenant les bras dans diverses

89. Waddington, Babelon et ReInACh 1925, p. 158, no 1.

90. WOJAN 2006, p. 188.

91. Dalaison 2008, p. 174-180 ; DaLAison 2010, p. 203-218.

92. Voir appendice et planche, figures 10-13.

93. Appien, Histoire de Mithridate, 12, 9, 66 et 12, 10, 70.

94. Strabon, Géographie, 15, 3, 13-15. 
positions ; une étoile. L'arbre, vraisemblablement un chêne, ainsi que l'aigle ${ }^{95}$ renvoient à Zeus ; le bœuf fait référence à un sacrifice offert à une divinité importante ; l'étoile est peut-être à mettre en relation avec les aspects iraniens de la divinité ${ }^{66}$; quant au quadrige, il symbolise le dieu suprême.

Zeus Stratios est une divinité complexe, qui s'est enrichie sur le long terme. Il a des origines pontiques, mélangées à des influences iraniennes (notamment par les apports du mazdéisme) et grecques. Au gré des vicissitudes historiques de la région, le dieu pontique a absorbé des éléments provenant d'autres divinités, fondamentalement assez proches de lui. Ces apports successifs l'ont étoffé et lui ont donné une personnalité composite. Zeus Stratios est la divinité pontique suprême, symbolisée par le quadrige dont les chevaux renvoient aux quatre éléments : l'eau, la terre, l'air et le feu. Ce dernier joue un rôle primordial dans le culte et se manifeste au moment de l'embrasement du bûcher. L'influence grecque se marque déjà dans la dénomination : comme cela s'observe pour la majorité des grands dieux indigènes, la divinité prend le nom de Zeus, dieu supérieur des Grecs, accompagné de l'épithète Stratios, qui signifie «guerrier». Il est d'ailleurs le seul, parmi les divinités que nous étudions dans cet article, à avoir changé de nom. Cela est peut-être à mettre en relation avec le fait que les Mithridatides, même s'ils se revendiquaient des origines iraniennes, ont cherché à se présenter comme des souverains hellénisés, reprenant à leur compte un certain nombre de pratiques gréco-macédoniennes ${ }^{97}$. L'hellénisation de la cour et des élites était relativement importante et l'on peut supposer que la terminologie grecque donnée au dieu souverain dans le royaume entrait dans le cadre de cette politique d'hellénisation. Zeus Stratios est donc le dieu suprême de la dynastie mithridatique, du royaume et des armées auxquels il apporte sa protection et son pouvoir. Il est en quelque sorte le successeur naturel du dieu iranien Ahura Mazda ou Ormuzd, souverain

95. À titre d'hypothèse, on pourrait se demander si l'aigle des monnaies d'Amaseia ne serait pas une sorte de déformation iconographique de la représentation traditionnelle d'Ahura-Mazda en Perse (un disque ailé d'où sort parfois un personnage barbu. Voir BRIANT 1996, p. 259-260 et fig. 28-29, qui rappelle en outre qu'Ahura-Mazda est aussi connu sous le nom de Zeus). De ce fait, l'aigle aurait une double signification : le renvoi aux aspects iraniens de la divinité honorée à Amaseia et l'évocation de son interpretatio graeca en Zeus Stratios.

96. L'étoile, qui peut également être interprétée comme un soleil (voir ici n. 71), est peut-être un symbole de Zeus Stratios assimilé à Ahura Mazda (même si à l'époque achéménide le culte du Soleil était semble-t-il séparé de celui d'Ahura Mazda - voir BRIANT 1996, p. 259-262 -, il y a eu une sorte d'assimilation entre Ahura Mazda, le Soleil et le Feu éternel aux périodes suivantes). Cependant, l'étoile - qui n'est figurée que sur des monnaies des premiers Sévères - apparaît également parfois sur des types de revers autres que ceux consacrés à Zeus Stratios. On la retrouve avec Tychè, Hadès-Sérapis, l'empereur faisant une libation, Caracalla et Géta se tenant la main. Dans les deux derniers cas, elle pourrait effectivement aussi renvoyer à Zeus Stratios, dans la mesure où, comme on le verra plus bas, le sanctuaire du dieu à Amaseia était également associé au culte impérial. En revanche, pour Tychè et Hadès-Sérapis, il est impossible d'établir une connexion directe avec Zeus Stratios. Il est donc difficile de déterminer exactement le sens de ce symbole et de le relier systématiquement à Zeus Stratios.

97. Callataÿ 2003, p. 220-222. 
du ciel et des hommes. La divinité pontique originelle s'est donc iranisée et hellénisée. Les changements ne portent pas seulement sur le nom et ne sont pas simplement un vernis superficiel. Ils concernent la nature même du dieu et ont contribué à l'enrichir au cours du temps, tout en lui gardant sa fonction initiale de dieu suprême.

Il faut ajouter quelques précisions en nous fondant sur un article récent de Stephen Mitchell ${ }^{98}$. En effet, les éléments découverts à Yassıçal ne sont pas antérieurs à la période romaine et Strabon ne mentionne à aucun moment ce site. Établissant un parallèle entre le fait que Yassiçal est un lieu de rassemblement de tous les habitants de la cité et le serment - dont le texte a été découvert à Vezirköprü (ancienne Neapolis) - prêté par tous les Paphlagoniens à l'empereur Auguste, Stephen Mitchell propose de voir un lien entre l'autel de Yassiçal et le culte impérial. Par ailleurs, il lui apparaît problématique de penser que ce site ait pu être consacré uniquement à Zeus Stratios, le dieu protecteur de Mithridate VI, grand ennemi des Romains. Ainsi, l'auteur suppose-t-il que, à la place d'une tradition religieuse soutenue par leur ennemi, les Romains ont introduit une nouvelle forme de rituel - le culte impérial - visant à prouver la fidélité des habitants d'Amaseia à l'empereur. Ce nouveau culte aurait quand même été pratiqué selon des rites locaux (et donc perses), afin d'être facilement accepté par la population. Cette interprétation est fort intéressante, d'autant qu' on pourrait voir dans le quadrige et l'aigle des monnaies des références à l'empereur. Néanmoins, ces nouveaux éléments ne suppriment pas le lien de Yassiçal avec Zeus Stratios, puisque le culte de ce dieu est attesté sur le site par deux inscriptions ${ }^{99}$. Le sanctuaire ne pouvait donc en aucun cas être uniquement consacré au culte impérial. Il est possible d'envisager qu'avant la période romaine, le culte de Zeus Stratios ait été rendu dans d'autres sites du royaume du Pont et qu'avec l'Empire, ait été créé à Yassiçal un nouveau sanctuaire public municipal qui associait Zeus Stratios, grand dieu régional - et désormais essentiellement poliade -, et le culte impérial ${ }^{100}$, qui s'installe dans les habits de ce très ancien culte pontique et réutilise la tradition perse de l'autel enflammé. La complexité et la richesse de la divinité n'ont donc pas disparu. Les divers éléments associés au bûcher sur les monnaies renvoient aux différentes composantes - et «périodes»-du dieu qui, à cette époque, sont toujours présentes dans Zeus Stratios, mais de façon si imbriquée qu'il ne semble pas réellement possible de les dissocier. Elles font partie intégrante du dieu et en sont l'essence même.

Comme souvent lorsqu'ils ont été confrontés aux divinités spécifiques des territoires qu'ils contrôlaient, les Romains ont fait preuve de pragmatisme. Du fait

98. Mitchell 2007b, p. 366-377.

99. Studia Pontica III, p. 150-152, nos 140-141.

100. On se retrouverait dans le même cas de figure qu'à Neocaesarea où Mèn et le culte impérial cohabitaient dans le même temple. Il s'agissait de valoriser l'empereur vis-à-vis des habitants de la cité en le plaçant dans le sanctuaire d'un dieu local prestigieux (il existe d'autres exemples de cette association en Asie Mineure : Çızmeli 2006, p. 123). 
de son nom grec et de ses fonctions proches de celles d'un Jupiter tout-puissant et même s'il possédait des attributions ancrées dans l'histoire religieuse locale, ils ont fait cohabiter Zeus Stratios et Jupiter dans le sanctuaire de Yassıçal par le biais du culte impérial. Désormais, le dieu était lié au nouveau pouvoir qui avait remplacé la dynastie mithridatique.

\section{Conclusion}

Sous les premiers Sévères, l'étude des revers monétaires des cités pontiques ne montre donc pas de grande nouveauté dans leur panthéon. Sur les six ateliers pontiques alors en activité (Amaseia, Comana Pontica, Neocaesarea, Trapezus, Zela et Sebastopolis), seul le dernier émet des types de revers exclusivement centrés sur son fondateur légendaire, Héraclès, alors que les cinq autres font référence dans leur monnayage à des divinités d'origine indigène ou iranienne. À la différence des autres dieux du panthéon gréco-romain «traditionnel» que l'on peut retrouver sur des monnaies de différents ateliers, ces dieux et ces déesses sont propres à chaque cité. Trois ont une origine indigène (Mâ, Mèn et Zeus Stratios), deux une origine iranienne (Anahita-Anaïtis et Mithra). Si Anahita-Anaïtis, Mithra et Zeus Stratios sont très présents dans le monnayage de leur cité et si Mâ est même la divinité exclusive des monnaies de Comana Pontica, les types consacrés à Mèn sont en revanche très secondaires à Neocaesarea. Pour autant, cela ne préjuge en rien de l'ancienneté du culte dans la cité, puisqu'il se pourrait - mais cela est loin d'être une certitude - que la présence de Mithra à Trapezus soit à dater de la période romaine. Quant aux quatre autres divinités, leur culte dans le Pont remonte à une «plus haute antiquité»; leur persistance et leur survivance sous les premiers Sévères ne sont pas liées à un quelconque regain d'intérêt pour un passé lointain, mais confirment au contraire l'importance de l'implantation et de l'influence indigènes et iraniennes dans le Pont ${ }^{101}$.

Si besoin était, c'est une confirmation des liens ancestraux du Pont avec le monde perse ${ }^{102}$ et de la permanence, au fil du temps, de cette influence ${ }^{103}$. La période de la dynastie des Mithridatides est marquée par cette iranisation,

101. Le langage iconographique des revers monétaires est largement connu et reconnu par les utilisateurs des pièces à l'époque romaine.

102. BRIANT 1996, p. 761-764.

103. Il existe une divinité, Baal Gazur, connue par des monnaies à légende araméenne du milieu du Ive siècle av. J.-C. émises à Gazioura - ville ayant disparu à l'époque romaine et initialement située sur l'Iris entre Amaseia et Comana Pontica. Ces pièces (WAdDInGton, BABElon, ReInACH 1925, p. 112-113, nos 1-4) ont été frappées sous le satrape Ariarathe, qui avait fait obédience aux Perses et s'était constitué un fief dans la région. Cet espace se situait aux marges de l'empire perse, puis de la conquête macédonienne, et a donc connu des vicissitudes avant de passer sous la domination des rois du Pont. Quoiqu'il en soit ce Baal Gazur «Seigneur de Gazioura», dont il ne semble subsister aucune trace à la période romaine, était sans doute un grand dieu local, particulièrement en faveur dans la satrapie d'Ariarathe, où les influences perses prédominaient. Voir OLSHAUSEN 1990, p. 1877 ; BRIANT 1996, p. 761-764. 
qui est d'ailleurs concurrencée et contrebalancée par la volonté d'hellénisation de la monarchie. En effet, comme le montre François de Callataÿ ${ }^{104}$, si les rois du Pont revendiquent des origines iraniennes (réelles ou supposées), ils ont cherché à se conduire en souverains hellénistiques en investissant dans des pratiques gréco-macédoniennes. Cette politique volontariste passe évidemment par l'emploi du grec dans les documents officiels - dont les monnaies -, mais également par la culture grecque, une politique de mariages, l'évergétisme, la présence de philosophes grecs à la cour, la fondation ou la refondation de cités au nom des souverains et enfin par la politique monétaire. Cette dernière a été examinée récemment par Christoph Michels ${ }^{105}$ dans le cadre d'une étude sur l'hellénisation des royaumes de Bithynie, du Pont et de Cappadoce. Si l'on s'en tient aux revers, les monnaies des rois du Pont représentent certes des dieux «grecs», comme Athéna et Nikè (sans doute sous Mithridate III), Zeus (sous Mithridate III, peut-être en lien avec Zeus Stratios), Zeus et Héra (Mithridate IV et Laodicè), Apollon (Mithridate V), mais elles font aussi une part aux cultes indigènes ou iraniens avec le symbole de l'étoile sur le croissant (qui apparaît dès Mithridate III) et la représentation de Mèn Pharnakou (Pharnace $\mathrm{I}^{\mathrm{er}}{ }^{106}$. À partir de Mithridate IV, la figure de Persée est très fréquente sur les monnaies. Ce dernier a l'avantage de réaliser une sorte de synthèse entre les origines iraniennes et les revendications grecques de la dynastie.

En effet, Persée, qui aurait donné son nom aux Perses, est en même temps un héros des Grecs. Le mythe de Persée est largement repris par Mithridate VI Eupator. Il apparaît à la fois sur les monnaies émises par les cités du royaume (où dominent également des figures de la mythologie grecque) et sur les monnaies royales. Sur ces dernières, avant la conquête de l'Asie par Mithridate, c'est Pégase - comme évocation de Persée - qui est représenté. Ensuite, il est supplanté par le cerf - et donc par Artémis, en lien avec Éphèse. À partir de ce moment-là, la propagande royale veut éliminer le côté achéménide de la royauté et montrer que Mithridate - comme Alexandre -, après avoir été le conquérant de la Grèce, en est désormais le bienfaiteur. Cependant, cette politique monétaire n'est pas forcément le reflet de la réalité, puisque le culte des divinités pontiques et iraniennes a continué à perdurer - et ce jusqu'à l'époque romaine. Les monnaies, qui circulent hors du Pont, sont censées être une sorte de «carte de visite» de la royauté qui veut essentiellement insister sur son aspect grec. Pourtant, dans la pratique de la cour, la réalité est plus nuancée et Mithridate continue d'honorer les divinités traditionnelles de son royaume, comme, par exemple, lorsqu'il sacrifie à Zeus Stratios ${ }^{107}$.

104. Callatä̈ 2003, p. 220-222.

105. Michels 2009, p. 183-219.

106. Voir ici n. 70 et 88.

107. Voir le témoignage d'Appien (ici n. 93). 
Au long des siècles, ces divinités d'origine indigène et iranienne ont profondément évolué. Leur culte est le résultat d'une histoire au cours de laquelle différentes «couches» se sont ajoutées et superposées pour aboutir à des figures complexes, qui, sous l'Empire romain, ne sont plus exactement identiques à ce qu'elles étaient aux origines. Pour autant, ces dieux gardent une partie de leur spécificité et il faut se méfier de certains auteurs ${ }^{108}$ qui ont tendance, parfois de manière artificielle, à vouloir voir partout du syncrétisme et à faire du panthéon une sorte de grand «fourre-tout» dans lequel il n'y aurait plus de distinction entre les divinités. Ces dieux ont parfois des points communs. Beaucoup de leurs cultes sont composites, hybrides et contiennent des éléments indigènes, iraniens et grecs, mais ils restent cependant distincts les uns des autres ${ }^{109}$.

Si nous admettons une assimilation organisée et volontaire des cités pontiques, comme des autres cités du monde romain, éventuellement avec des variantes liées à certains territoires précis, il faut aussi admettre que, sous l'Empire, il n'exista bientôt plus de différence entre des dieux qui seraient gréco-romains et des dieux qui seraient restés indigènes. Ils sont tous un peu des deux. Les divinités honorées ne sont pas de simples transpositions de dieux superficiellement grécisés, voire romanisés, car remplacer le nom indigène d'un dieu par un nom grec (AnahitaAnaïtis) - ou même lui accoler une épithète grecque (Zeus Stratios) - indique des permanences qui s'expriment dans des langages différents selon les époques. Étant donné que les divinités indigènes pouvaient avoir en fait des personnalités et des fonctions très proches de celles des dieux des Grecs et des Romains, ne serait-ce que par leurs origines indo-européennes communes, elles ont pu être «interprétées » et conserver une certaine dimension locale. Comme leurs dévots, elles s'étaient grécisées, puis romanisées. Pour les cultores d'une religion polythéiste, il n'y a pas différents «niveaux» de dieux : les divinités se rajoutent et se complètent. Ils les honorent en fonction de leurs besoins propres et des fonctions des diverses divinités, mais ils vénèrent aussi, et peut-être surtout, les dieux de la cité qui assurent la protection de toute la communauté.

108. Notamment, SAPRYKIN 2009,p. 258-260. L'auteur écrit que Zeus était un dieu multifonctionnel et voit des liens entre Zeus, Mèn Pharnakou, Mithra, Persée et Dionysos...

109. Olshausen 1990, p. 1904-1906 ; Mitchell 2007a, p. 169-171. 


\section{APPENDICE}

Tableaux récapitulatifs des types de revers retenus

\section{Anahita-Anaïtis / Zela ${ }^{110}$}

\begin{tabular}{|l|c|l|l|l|}
\hline Empereur & Date & Description du type de revers & Légende & Références \\
\hline Caracalla & $205 / 206$ & $\begin{array}{l}\text { Anahita-Anaïtis, drapée, la tête voilée, debout } \\
\text { de face, tenant un épi dans la main droite et } \\
\text { un objet indistinct dans la main gauche }\end{array}$ & $\begin{array}{l}\text { ZHLIT [ ] ONT } ; \\
\text { à l'exergue : ET PMB }\end{array}$ & R 41, no 44 \\
\hline
\end{tabular}

\section{Mâ/Comana Pontica}

\begin{tabular}{|c|c|c|c|c|}
\hline Empereur & Date & Description du type de revers & Légende & Références \\
\hline \multirow[t]{7}{*}{ Septime Sévère } & $205 / 206$ & $\begin{array}{l}\text { Mâ debout de face, la main droite reposant sur } \\
\text { un bouclier, tenant de la main gauche une massue } \\
\text { appuyée contre son épaule dans la cella centrale } \\
\text { d'un temple tétrastyle ; dans le fronton, aigle } \\
\text { luttant avec un serpent }\end{array}$ & $\begin{array}{l}\text { IEPOKAICA-KOMA } \\
\text { NESN; } \\
\text { à l'exergue : ET BOP }\end{array}$ & R 15, no 15 \\
\hline & $205 / 206$ & $\begin{array}{l}\text { Mâ debout de face, la main droite reposant sur } \\
\text { un bouclier, tenant de la main gauche une massue } \\
\text { appuyée contre son épaule dans la cella centrale } \\
\text { d'un temple tétrastyle ; dans le fronton, disque }\end{array}$ & $\begin{array}{l}\text { IEPOKAICA-KOMA NE ; } \\
\text { à l'exergue : ET BOP }\end{array}$ & R 16, no 16 \\
\hline & $205 / 206$ & $\begin{array}{l}\text { Mâ, tête radiée, vêtue de la stola, debout de } \\
\text { face, la main droite reposant sur un bouclier, } \\
\text { tenant de la main gauche une massue appuyée } \\
\text { contre son épaule }\end{array}$ & $\begin{array}{l}\text { IEPOKAICA-KOMA } \\
\text { NESN; } \\
\text { à l'exergue : ET BOP }\end{array}$ & R $17, n^{\text {os }} 17-18$ \\
\hline & $205 / 206$ & $\begin{array}{l}\text { temple tétrastyle à fronton échancré ; dans la cella } \\
\text { centrale, bétyle de Mâ surmonté d'une Victoire } \\
\text { à droite, tenant une couronne et une palme }\end{array}$ & $\begin{array}{l}\text { IEPOKAICA-KOMA } \\
\text { NESN ; } \\
\text { à l'exergue : ET BOP }\end{array}$ & R 19, no20 \\
\hline & $205 / 206$ & $\begin{array}{l}\text { bétyle de Mâ dont le corps cylindrique est } \\
\text { entouré de guirlandes portées par des figures } \\
\text { féminines; sur ce corps s'élève un dôme } \\
\text { flanqué de deux anneaux de portage, lui-même } \\
\text { surmonté d'une Victoire à droite tenant une } \\
\text { couronne et une palme }\end{array}$ & $\begin{array}{l}\text { IEPOKAICA-KOMA } \\
\text { NESN; } \\
\text { à l'exergue : ET BOP }\end{array}$ & R $20, n^{\circ} 21$ \\
\hline & $205 / 206$ & $\begin{array}{l}\text { temple tétrastyle ; dans la cella centrale, bétyle } \\
\text { de Mâ surmonté d'une Victoire à droite, tenant } \\
\text { une couronne et une palme }\end{array}$ & $\begin{array}{l}\text { IEPOKAICA-KO-MANE } \Omega, \\
\text { avec des variantes ; } \\
\text { à l'exergue : ET BOP } \\
\end{array}$ & $\begin{array}{l}\text { R 23-37, } \\
\text { nos 24-38 }\end{array}$ \\
\hline & $205 / 206$ & $\begin{array}{l}\text { temple tétrastyle ; dans la cella centrale, bétyle } \\
\text { de Mâ surmonté d'une Victoire à gauche, tenant } \\
\text { une couronne et une palme }\end{array}$ & $\begin{array}{l}\text { IEPOKAICA-KO-MANE; } \\
\text { à l'exergue : ET BOP }\end{array}$ & $\begin{array}{l}\text { R 38-41, } \\
\text { nos 39-41 }\end{array}$ \\
\hline
\end{tabular}

110. Nous n'avons pas retenu le temple hexastyle à fronton échancré au milieu (?), sans divinité, des émissions de Septime Sévère, Iulia Domna, Caracalla et Géta (R 14-16, nos 77, 81, 82 ; R 26-27, nos 36-37 ; R 30-31, nos 30-31 ; R 35-41, nos 38-44...), car il n'est pas sûr que ce soit le temple d'Anahita. 


\begin{tabular}{|c|c|c|c|c|}
\hline Empereur & Date & Description du type de revers & Légende & Références \\
\hline \multirow[t]{3}{*}{ Caracalla } & $205 / 206$ & $\begin{array}{l}\text { temple tétrastyle ; dans la cella centrale, bétyle } \\
\text { de Mâ surmonté d'une Victoire à droite, tenant } \\
\text { une couronne et une palme ; dans le fronton, } \\
\text { disque }\end{array}$ & $\begin{array}{l}\text { IEPOKAICA-KO-MANE, } \\
\text { avec des variantes ; } \\
\text { à l'exergue : ET BOP }\end{array}$ & $\begin{array}{l}\text { R } 31, \text { no } 48 \\
\text { R } 34, \text { no } 49 \\
\text { R } 35, \text { nos } 54-55 \\
\text { R } 42, \text { no } 45 \\
\text { R } 43, \text { no } 46 \\
\text { R } 44, \text { no } 47 \\
\text { R } 45, \text { no } 50 \\
\text { R } 46, \text { no } 51 \\
\text { R } 47, \text { no } 52 \\
\text { R } 48, \text { no } 53\end{array}$ \\
\hline & $205 / 206$ & $\begin{array}{l}\text { temple tétrastyle ; dans la cella centrale, bétyle } \\
\text { de Mâ surmonté d'une Victoire à gauche, tenant } \\
\text { une couronne et une palme; dans le fronton, } \\
\text { disque }\end{array}$ & $\begin{array}{l}\text { IEPOKAICA-KO-MANE, } \\
\text { avec des variantes ; } \\
\text { à l'exergue : ET BOP }\end{array}$ & $\begin{array}{l}\text { R } 40, \text { no } 56 \\
\text { R } 49, \text { no } 57 \\
\text { R } 50, \text { no } 58\end{array}$ \\
\hline & $205 / 206$ & $\begin{array}{l}\text { Mâ (?) debout à gauche sur une estrade, tenant } \\
\text { une massue (?) et un arc (?) }\end{array}$ & $\begin{array}{l}\text { IEPOKAICA-KOMA } \\
\text { NE } \Omega \text {; } \\
\text { sur l'estrade : ET BOP }\end{array}$ & $\mathrm{R} 51, \mathrm{n}^{\circ} 60$ \\
\hline \multirow[t]{2}{*}{ Géta } & $205 / 206$ & $\begin{array}{l}\text { temple tétrastyle; dans la cella centrale, bétyle } \\
\text { de Mâ surmonté d'une Victoire à droite, tenant } \\
\text { une couronne et une palme; dans le fronton, } \\
\text { disque }\end{array}$ & $\begin{array}{l}\text { IEPOKAICA-KO-MANE ; } \\
\text { à l'exergue : ET BOP }\end{array}$ & $\begin{array}{l}\text { R } 32, \mathrm{n}^{\circ} 61 \\
\text { R } 52, \mathrm{n}^{\circ} 62\end{array}$ \\
\hline & $205 / 206$ & $\begin{array}{l}\text { temple tétrastyle; dans la cella centrale, bétyle } \\
\text { de Mâ surmonté d'une Victoire à gauche, } \\
\text { tenant une couronne et une palme; dans le } \\
\text { fronton, disque }\end{array}$ & $\begin{array}{l}\text { IEPOKAICA-KO-MANE ; } \\
\text { à l'exergue : ET BOP }\end{array}$ & $\mathrm{R} 40, \mathrm{n}^{\mathrm{o}} 63$ \\
\hline
\end{tabular}

\section{Mèn/Neocaesarea}

\begin{tabular}{|l|c|l|l|l|}
\hline Empereur & Date & Description du type de revers & Légende & Références \\
\hline Géta César & $205 / 206$ & $\begin{array}{l}\text { Tychè coiffée du calathos, assise à gauche, } \\
\text { tenant une corne d'abondance dans la main } \\
\text { droite et un sceptre dans la main gauche, } \\
\text { couronnée par l'empereur (?) debout à gauche, } \\
\text { tenant un sceptre dans la main gauche ; devant } \\
\text { eux, Mèn debout à droite, tenant un sceptre } \\
\text { dans la main gauche }\end{array}$ & $\begin{array}{l}\text { MHTO ; ET P[ ] } \\
\text { à 22, no 211 }\end{array}$ & \\
\hline
\end{tabular}

\section{Mithra/Trapezos}

\begin{tabular}{|c|c|c|c|c|}
\hline Empereur & Date & Description du type de revers & Légende & Références \\
\hline Septime Sévère & 194-195 & $\begin{array}{l}\text { Mithra à cheval à droite, entre un arbre } \\
\text { et un autel }\end{array}$ & $\begin{array}{l}\text { ТРАПЕ[ ]; } \\
\text { entre les jambes du cheval : } \\
\text { А- } \Lambda \Pi\end{array}$ & R 29, nos 33-34 \\
\hline \multirow[t]{2}{*}{ Iulia Domna } & 194-195 & $\begin{array}{l}\text { Mithra à cheval à droite, entre un arbre } \\
\text { et un autel }\end{array}$ & $\begin{array}{l}\text { ТРАПЕ[ ]; } \\
\text { entre les jambes du cheval : } \\
\text { А- } \Lambda \Pi \text {, avec des variantes }\end{array}$ & $\begin{array}{l}\text { R } 29, \text { no } 36 \\
\text { R } 31, \text { no } 37 \\
\text { R } 32, \text { no } 38\end{array}$ \\
\hline & 216-217 & $\begin{array}{l}\text { Mithra à cheval à droite ; derrière un arbre; } \\
\text { devant une colonne au sommet de laquelle } \\
\text { se trouve un oiseau (corbeau?) }\end{array}$ & $\begin{array}{l}\text { TPAП-EZOYNTI- } \Omega N \text {; } \\
\text { à l'exergue : ET PNГ }\end{array}$ & R 45, no 52 \\
\hline
\end{tabular}




\begin{tabular}{|c|c|c|c|c|}
\hline Empereur & Date & Description du type de revers & Légende & Références \\
\hline \multirow[t]{5}{*}{ Caracalla } & 198-199 & $\begin{array}{l}\text { buste drapé de Mithra à droite, vu de trois quarts } \\
\text { en arrière, coiffé du bonnet phrygien radié ; } \\
\text { devant, le protomé de son cheval }\end{array}$ & $\begin{array}{l}{[\quad] Y N-T I \Omega N \text { ETOYC ; }} \\
\text { en dessous : E } \Lambda \Pi \text {, } \\
\text { avec des variantes }\end{array}$ & $\begin{array}{l}\text { R } 35, n^{\circ} 41 \\
\text { R } 36, n^{\circ} 42\end{array}$ \\
\hline & $216-217$ & $\begin{array}{l}\text { Mithra à cheval à droite, } \\
\text { entre un arbre et un autel }\end{array}$ & $\begin{array}{l}\text { TРАП[ ] ; } \\
\text { à l'exergue : ET PNГ }\end{array}$ & R 39, no45 \\
\hline & $216-217$ & Mithra à cheval à droite ; devant, un autel & $\begin{array}{l}\text { TPAПEZO-NTI } \Omega N \text {; } \\
\text { à l'exergue : ET PNГ, } \\
\text { avec des variantes }\end{array}$ & $\begin{array}{l}\text { R } 40, \text { no } 46 \\
R \quad 41, \text { no47 }\end{array}$ \\
\hline & $216-217$ & $\begin{array}{l}\text { Mithra à cheval à droite; derrière, un arbre et } \\
\text { Cautès abaissant sa torche; devant, un autel et } \\
\text { une colonne, au sommet de laquelle se trouve } \\
\text { un oiseau (corbeau ?) et Cautopatès levant } \\
\text { sa torche }\end{array}$ & $\begin{array}{l}\text { TPAПEZOYNTI } \Omega N \text {; } \\
\text { à l'exergue : ET PNГ }\end{array}$ & $\mathrm{R} 42, \mathrm{n} \times 48$ \\
\hline & $216-217$ & $\begin{array}{l}\text { Mithra à cheval à droite; derrière, un arbre; } \\
\text { devant, un autel et une colonne, au sommet de } \\
\text { laquelle se trouve un oiseau (corbeau?) }\end{array}$ & $\begin{array}{l}\text { [ ] ; } \\
\text { à l'exergue : ET PNГ }\end{array}$ & $\mathrm{R} 43, \mathrm{n}$ os $49-50$ \\
\hline Géta & $198-199$ & $\begin{array}{l}\text { Mithra à cheval à droite, } \\
\text { entre un arbre et un autel }\end{array}$ & [ ]ZOYN[ ] & $\mathrm{R} 33, \mathrm{n} \times 39$ \\
\hline
\end{tabular}

\section{Zeus Stratios / Amaseia}

\begin{tabular}{|c|c|c|c|c|}
\hline Empereur & Date & Description du type de revers & Légende & Références \\
\hline \multirow[t]{6}{*}{ Septime Sévère } & $205 / 206$ & $\begin{array}{l}\text { bûcher enflammé à un étage ; } \\
\text { à gauche un arbre }\end{array}$ & $\begin{array}{l}\text { A } \triangle \mathrm{P} \text { CE ANT AMACIAC } \\
\text { MHT NE ПP PON ; } \\
\text { à l'exergue : ET CH }\end{array}$ & $\begin{array}{l}\text { R } 194, \text { no } 240 \\
\text { R } 195, \text { no } 241 \\
\text { R } 196, \text { no } 242\end{array}$ \\
\hline & $205 / 206$ & $\begin{array}{l}\text { bûcher enflammé à deux étages ; } \\
\text { à gauche un arbre }\end{array}$ & $\begin{array}{l}\text { A } \triangle P \text { CEY ANT AMACIAC } \\
\text { MH NE } \Pi \text {, } \\
\text { avec des variantes ; } \\
\text { à l'exergue : ET CH }\end{array}$ & $\begin{array}{l}\text { R } 197, n^{\circ} 243 \\
\text { R } 198, \text { no } 244 \\
\text { R } 199, \text { no } 245 \\
\text { R 200, no } 246 \\
\text { R 202, no } 248 \\
\text { R 203, no } 249 \\
\text { R 204, no } 250 \\
\text { R 205, no } 251 \\
\text { R 206, no } 252 \\
\text { R 207, no } 253\end{array}$ \\
\hline & $205 / 206$ & $\begin{array}{l}\text { bûcher enflammé à deux étages; à gauche } \\
\text { un arbre ; objet indistinct sur le bûcher à droite }\end{array}$ & $\begin{array}{l}\text { A } \triangle \mathrm{P} \text { CEY ANT AMACIA } \\
\text { MH NE ПP } \Pi \text {; } \\
\text { à l'exergue : ET CH }\end{array}$ & R 201, no $^{\circ} 247$ \\
\hline & $205 / 206$ & $\begin{array}{l}\text { bûcher à un étage sur lequel se trouve un aigle } \\
\text { éployé de face, la tête levée, tenant une couronne } \\
\text { dans son bec ; à gauche, un arbre }\end{array}$ & $\begin{array}{l}\text { A } \triangle \text { CEY ANT AMACIAC } \\
\text { MHT NE ПР ПO, } \\
\text { avec des variantes ; } \\
\text { à l'exergue : ET CH }\end{array}$ & $\begin{array}{l}\text { R } 208, \text { no } 254 \\
\text { R } 215, \text { no } 261\end{array}$ \\
\hline & $205 / 206$ & $\begin{array}{l}\text { bûcher à un étage sur lequel se trouve un aigle } \\
\text { éployé de face, la tête à gauche, tenant une } \\
\text { couronne dans son bec; à gauche, un arbre }\end{array}$ & $\begin{array}{l}\text { A } \triangle \text { CEY ANT AMAC } \\
\text { MHT NE ПP ПON, } \\
\text { avec des variantes ; } \\
\text { à l'exergue : ET CH }\end{array}$ & $\begin{array}{l}\text { R } 209, n^{\circ} 255 \\
\text { R } 210, n^{\circ} 256 \\
\text { R212, no } 258 \\
\text { R } 213, n^{\circ} 259 \\
\text { R } 217, n^{\circ} 263\end{array}$ \\
\hline & $205 / 206$ & $\begin{array}{l}\text { bûcher à un étage sur lequel se trouve un aigle } \\
\text { éployé de face, la tête à gauche, tenant une } \\
\text { couronne dans son bec; à gauche, un arbre. } \\
\text { Objet indistinct sur le bûcher à droite (aigle } \\
\text { ou branche dans un cercle de grènetis ?) }\end{array}$ & $\begin{array}{l}\text { A } \triangle \text { CEY ANT AMACIAC } \\
\text { MHT NE ПP П ; } \\
\text { à l'exergue : ET CH }\end{array}$ & R 220, no 266 \\
\hline
\end{tabular}




\begin{tabular}{|c|c|c|c|c|}
\hline Empereur & Date & Description du type de revers & Légende & Références \\
\hline \multirow[t]{8}{*}{$\begin{array}{l}\text { Septime Sévère } \\
\text { (suite) }\end{array}$} & $205 / 206$ & $\begin{array}{l}\text { bûcher à un étage sur lequel se trouve un aigle } \\
\text { éployé de face, la tête à droite, tenant une cou- } \\
\text { ronne dans son bec ; à gauche, un arbre }\end{array}$ & $\begin{array}{l}\text { A } \triangle \text { CEY ANT AMACIAC } \\
\text { MHT NEOKE ПO, } \\
\text { avec des variantes; } \\
\text { à l'exergue : ET CH }\end{array}$ & $\begin{array}{l}\text { R } 211, \text { no } 257 \\
\text { R } 214, \text { no } 260 \\
\text { R } 216, \text { no } 262 \\
\text { R } 218, \text { no } 264\end{array}$ \\
\hline & $205 / 206$ & $\begin{array}{l}\text { bûcher à un étage sur lequel se trouve un aigle } \\
\text { éployé de face, la tête à droite (?), tenant une } \\
\text { couronne dans son bec (?); à gauche, un arbre; } \\
\text { objet indéterminé sous la date }\end{array}$ & $\begin{array}{l}\text { A } \triangle \text { CEY AN [ ] ACIAC } \\
\text { MH NE ПP; } \\
\text { à l'exergue : ET CH }\end{array}$ & R 219, no 265 \\
\hline & $205 / 206$ & $\begin{array}{l}\text { bûcher à un étage sur lequel se trouve un bœuf } \\
\text { couché sur le dos; à gauche, un arbre }\end{array}$ & $\begin{array}{l}\text { A } \triangle \mathrm{P} \text { CE ANT AMACIAC } \\
\text { MHT NE ПР ПO ; } \\
\text { à l'exergue : ET CH }\end{array}$ & R 221, no 267 \\
\hline & $205 / 206$ & $\begin{array}{l}\text { bûcher à un étage ; à gauche, un arbre ; sur le } \\
\text { bûcher Hélios dans un quadrige de face, le bras } \\
\text { droit levé. Sans aigle entre le bûcher et le } \\
\text { quadrige (?) }\end{array}$ & $\begin{array}{l}\text { [ ]Y ANT AMACIAC } \\
\text { MH [ ] ПO ; } \\
\text { à l'exergue : ET CH }\end{array}$ & R 222, no $^{\circ} 268$ \\
\hline & $205 / 206$ & $\begin{array}{l}\text { bûcher à un étage ; à gauche, un arbre ; sur le } \\
\text { bûcher Hélios dans un quadrige de face, le bras } \\
\text { droit levé, un fouet dans la main gauche }\end{array}$ & $\begin{array}{l}\text { A } \triangle \text { P CEY ANT AMACI } \\
\text { MH NE ПР ПO ; } \\
\text { dans le champ : ET CH }\end{array}$ & R 223, no 269 \\
\hline & $205 / 206$ & $\begin{array}{l}\text { bûcher à un étage ; à gauche, un arbre ; sur le } \\
\text { bûcher une pyramide ; au-dessus Hélios dans } \\
\text { un quadrige formé de deux couples de chevaux } \\
\text { superposés dont on ne voit que la partie anté- } \\
\text { rieure de profil }\end{array}$ & $\begin{array}{l}\text { [ ]; } \\
\text { à l'exergue : [ ] CH }\end{array}$ & R 224, no 270 \\
\hline & $205 / 206$ & $\begin{array}{l}\text { bûcher à un étage ; à gauche, un arbre ; sur } \\
\text { le bûcher un aigle éployé de face, la tête à } \\
\text { gauche ; au-dessus, Hélios dans un quadrige de } \\
\text { face, le bras droit levé, un fouet dans la main } \\
\text { gauche }\end{array}$ & $\begin{array}{l}\text { A } \triangle \text { CEY ANT AMACIIAC } \\
\text { MHT NE ПO ; } \\
\text { à l'exergue : ET CH }\end{array}$ & $\begin{array}{l}\text { R } 225, \text { no } 271 \\
\text { R } 226, \text { no } 272\end{array}$ \\
\hline & $206 / 207$ & $\begin{array}{l}\text { bûcher à un étage ; à gauche, un arbre ; sur } \\
\text { le bûcher un aigle éployé de face, la tête à } \\
\text { gauche ; au-dessus, Hélios dans un quadrige de } \\
\text { face ; à l'exergue, un bœuf couché sur le dos ; } \\
\text { une étoile dans le champ à gauche }\end{array}$ & $\begin{array}{l}\text { A } \triangle \text { P CEY AN AMACIAC } \\
\text { MHT NE } \Pi \text {; } \\
\text { dans le champ : ET C } \Theta\end{array}$ & R 247, no 296 \\
\hline $\begin{array}{l}\text { Septime Sévère } \\
\text { ou Caracalla }\end{array}$ & $206 / 207$ & $\begin{array}{l}\text { bûcher à un étage sur lequel se trouve un aigle } \\
\text { éployé de face, la tête à gauche tenant une cou- } \\
\text { ronne dans son bec ; à gauche, un arbre ; sur le } \\
\text { bûcher, objets indistincts (branches ?) à gauche } \\
\text { et à droite de l'aigle (?) }\end{array}$ & $\begin{array}{l}\text { A } \triangle \text { P CEY ANT AMACIAC } \\
\text { MH NE ПP П ; } \\
\text { à l'exergue : ET C } \Theta\end{array}$ & R 408, no 536 \\
\hline Iulia Domna & $205 / 206$ & $\begin{array}{l}\text { bûcher enflammé à deux étages ; } \\
\text { à gauche un arbre }\end{array}$ & $\begin{array}{l}\text { A } \triangle \text { P CEY ANT AMACIAC } \\
\text { MHT NE ПР П, } \\
\text { avec des variantes ; } \\
\text { à l'exergue : ET CH }\end{array}$ & $\begin{array}{l}\text { R } 252, \text { no } 301 \\
\text { R } 253, \text { no } 302 \\
\text { R } 254, \text { no } 303 \\
\text { R } 255, \text { no } 304 \\
\text { R } 256, \text { no } 305 \\
\text { R } 257, \text { no } 306\end{array}$ \\
\hline \multirow[t]{2}{*}{ Caracalla } & $205 / 206$ & $\begin{array}{l}\text { bûcher enflammé à un étage ; } \\
\text { à gauche un arbre }\end{array}$ & $\begin{array}{l}\text { A } \triangle \text { CEY ANT AMACIAC } \\
\text { MHT NE ПP, } \\
\text { avec des variantes ; } \\
\text { à l'exergue : ET CH }\end{array}$ & $\begin{array}{l}\text { R } 299, \text { no } 366 \\
\text { R } 300, \text { no } 367 \\
\text { R } 301, \text { no } 368 \\
\text { R } 303 \text {, no } 370 \\
\text { R } 304, \text { nos } 371- \\
372\end{array}$ \\
\hline & $205 / 206$ & $\begin{array}{l}\text { bûcher enflammé à un étage ; à gauche un } \\
\text { arbre; sur le bûcher, des branches à gauche et } \\
\text { à droite des flammes }\end{array}$ & $\begin{array}{l}\text { A } \triangle \text { P CEY ANT AMACIAC } \\
\text { MHT NE ПP ; } \\
\text { à l'exergue : ET CH }\end{array}$ & R 298, no 365 \\
\hline
\end{tabular}




\begin{tabular}{|c|c|c|c|c|}
\hline Empereur & Date & Description du type de revers & Légende & Références \\
\hline \multirow[t]{10}{*}{$\begin{array}{l}\text { Caracalla } \\
\text { (suite) }\end{array}$} & $205 / 206$ & $\begin{array}{l}\text { bûcher enflammé à un étage ; à gauche un } \\
\text { arbre ; sur le bûcher, des branches à gauche et } \\
\text { à droite des flammes ; traces au sommet du } \\
\text { bûcher (partie antérieure d'un bœuf à droite?) }\end{array}$ & $\begin{array}{l}\text { A } \triangle \mathrm{P} \text { CE ANT ACIAC } \\
\text { MHT NE ПP ПONTO } \\
\text { à l'exergue : ET CH }\end{array}$ & R 302, no 369 \\
\hline & $205 / 206$ & $\begin{array}{l}\text { bûcher enflammé à deux étages ; } \\
\text { à gauche un arbre }\end{array}$ & $\begin{array}{l}\text { A } \triangle \text { P CEY ANT AMACIAC } \\
\text { MH NE } \Pi \text { П, } \\
\text { avec des variantes ; } \\
\text { à l'exergue : ET CH }\end{array}$ & 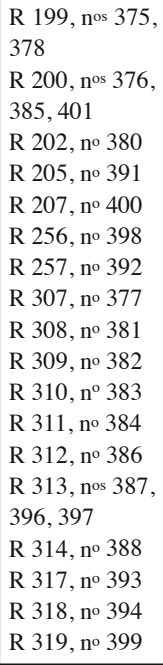 \\
\hline & $205 / 206$ & $\begin{array}{l}\text { bûcher enflammé à deux étages ; à gauche un } \\
\text { arbre ; objet indistinct sur le bûcher à droite }\end{array}$ & $\begin{array}{l}\text { A } \triangle \text { P CEY ANT AMACIAC } \\
\text { MHT NE } \Omega \text { ПР ПO ; } \\
\text { à l'exergue : ET CH }\end{array}$ & R 201, no 379 \\
\hline & $205 / 206$ & $\begin{array}{l}\text { bûcher enflammé à deux étages ; à gauche un } \\
\text { arbre; sur le bûcher, une branche à gauche } \\
\text { des flammes }\end{array}$ & $\begin{array}{l}\text { A } \triangle \text { P CEY ANT AMACIAC } \\
\text { MHT NE ПP ; } \\
\text { à l'exergue : ET CH }\end{array}$ & R 316, no 390 \\
\hline & $205 / 206$ & $\begin{array}{l}\text { bûcher à un étage sur lequel se trouve un aigle } \\
\text { éployé de face, la tête à droite tenant une } \\
\text { couronne dans son bec; à gauche, un arbre }\end{array}$ & $\begin{array}{l}\text { A } \triangle \text { P CEY ANT AMACIAC } \\
\text { MH NE } \Pi \text { П ; } \\
\text { à l'exergue : ET CH }\end{array}$ & R 320, no 402 \\
\hline & $205 / 206$ & $\begin{array}{l}\text { bûcher à un étage sur lequel se trouve un aigle } \\
\text { éployé de face, la tête à droite tenant une } \\
\text { couronne dans son bec (?) ; à droite, un arbre }\end{array}$ & $\begin{array}{l}\mathrm{A} \triangle \mathrm{P} \mathrm{C}[\mathrm{N} \text { ] } \Omega K ~ \Pi P \\
\mathrm{T} \Pi \mathrm{\Pi ON} ; \\
\text { à l'exergue : ET C[ ] }\end{array}$ & R 323 , no 407 \\
\hline & $205 / 206$ & $\begin{array}{l}\text { bûcher à un étage sur lequel se trouve un aigle } \\
\text { éployé de face, la tête à gauche, tenant une } \\
\text { couronne dans son bec; à gauche, un arbre. } \\
\text { Objet indistinct sur le bûcher à droite (aigle ou } \\
\text { branche dans un cercle de grènetis?) }\end{array}$ & $\begin{array}{l}\text { A } \triangle P \text { CEY ANT AMACIAC } \\
\text { MHT NE ПP П ; } \\
\text { à l'exergue : ET CH }\end{array}$ & $\begin{array}{l}\text { R 220, } \\
\text { nos } 403,405\end{array}$ \\
\hline & $205 / 206$ & $\begin{array}{l}\text { bûcher enflammé à deux étages; à gauche, } \\
\text { un arbre; entre les deux étages du bûcher, } \\
\text { une guirlande }\end{array}$ & $\begin{array}{l}\text { A } \triangle \text { CE AN AMACIAC } \\
\text { MHT; } \\
\text { à l'exergue : ET CH }\end{array}$ & R 315, no 389 \\
\hline & $205 / 206$ & $\begin{array}{l}\text { bûcher à un étage sur lequel se trouve un aigle } \\
\text { éployé de face, la tête à gauche tenant une } \\
\text { couronne dans son bec; à gauche, un arbre }\end{array}$ & $\begin{array}{l}\text { A } \triangle \text { P CEY ANT AMACIAC } \\
\text { MHT NE ПP ПO, avec des } \\
\text { variantes ; } \\
\text { dans le champ : ET CH }\end{array}$ & $\begin{array}{l}\text { R } 321, \text { no } 404 \\
\text { R } 322, \text { no } 406\end{array}$ \\
\hline & $205 / 206$ & $\begin{array}{l}\text { bûcher à un étage sur lequel se trouve un aigle } \\
\text { éployé de face, la tête à droite (?), sous un } \\
\text { fronton; à gauche, un arbre }\end{array}$ & $\begin{array}{l}\mathrm{A} \triangle \mathrm{P} \text { CE ANT AM [ ] } \\
\text { à l'exergue : ET CH }\end{array}$ & R 324, no 408 \\
\hline
\end{tabular}




\begin{tabular}{|c|c|c|c|c|}
\hline Empereur & Date & Description du type de revers & Légende & Références \\
\hline \multirow[t]{12}{*}{$\begin{array}{l}\text { Caracalla } \\
\text { (suite) }\end{array}$} & $205 / 206$ & $\begin{array}{l}\text { bûcher à un étage ; à gauche, un arbre ; sur le } \\
\text { bûcher un bœuf couché sur le dos ; au-dessus, } \\
\text { un aigle éployé de face, la tête à gauche, tenant } \\
\text { une couronne dans son bec }\end{array}$ & $\begin{array}{l}\text { A } \triangle \mathrm{P} C E Y \text { ANT AMACIAC } \\
\text { MH NE } \Pi \text {; } \\
\text { à l'exergue : ET CH }\end{array}$ & $\begin{array}{l}\text { R } 325, \\
\text { nos } 409,410\end{array}$ \\
\hline & $205 / 206$ & $\begin{array}{l}\text { bûcher à un étage ; à gauche, un arbre ; sur le } \\
\text { bûcher une pyramide ; au-dessus, Hélios dans } \\
\text { un quadrige de face, le bras droit levé, un fouet } \\
\text { dans la main gauche }\end{array}$ & $\begin{array}{l}\text { A } \triangle P \text { CE AN AMAC } \\
\text { MH NE ПP ; } \\
\text { à l'exergue : ET CH }\end{array}$ & R 326 , no 411 \\
\hline & $205 / 206$ & $\begin{array}{l}\text { bûcher enflammé à deux étages sur lequel se } \\
\text { trouve un bœuf couché sur le dos; à gauche } \\
\text { un arbre }\end{array}$ & $\begin{array}{l}\text { A } \triangle \text { CE ANT AMACIAC } \\
\text { MHT NE ПP ПO ; } \\
\text { à l'exergue : ET CH }\end{array}$ & R 327, no 412 \\
\hline & $206 / 207$ & $\begin{array}{l}\text { bûcher enflammé à un étage ; } \\
\text { à gauche un arbre }\end{array}$ & $\begin{array}{l}\text { A } \triangle \text { P CEY ANT AMACIAC } \\
\text { MHT NE ПP ; } \\
\text { à l'exergue : ET C } \Theta\end{array}$ & R 397, no $^{\circ} 506$ \\
\hline & $206 / 207$ & $\begin{array}{l}\text { bûcher enflammé à deux étages ; } \\
\text { à gauche un arbre }\end{array}$ & $\begin{array}{l}\text { A } \triangle P \text { CE AN AMACIAC } \\
\text { MH NE ПP ПO, } \\
\text { avec des variantes ; } \\
\text { à l'exergue : ET C } \Theta\end{array}$ & $\begin{array}{l}\text { R } 243, \text { n० } 507 \\
\text { R } 244, \text { no } 508 \\
\text { R } 398, \text { no } 509 \\
\text { R } 399, \text { no } 510 \\
\text { R } 400, \text { no }^{\circ} 511 \\
\text { R } 401, \text { no }^{\circ} 512 \\
\text { R } 402, \text { no }^{\circ} 513 \\
\text { R } 403, \text { no } 514 \\
\text { R } 404, \text { nos } 515, \\
518\end{array}$ \\
\hline & $206 / 207$ & $\begin{array}{l}\text { bûcher enflammé à deux étages ; à gauche } \\
\text { un arbre; sur le premier étage du bûcher, des } \\
\text { branches à gauche et à droite du second étage }\end{array}$ & $\begin{array}{l}\text { A } \triangle \text { P CEY ANT AMACIAC } \\
\text { MHT NE П П ; } \\
\text { à l'exergue : ET C } \Theta\end{array}$ & R 405, no $^{\circ} 516$ \\
\hline & $206 / 207$ & $\begin{array}{l}\text { bûcher enflammé à deux étages ; à gauche } \\
\text { un arbre ; une étoile dans le champ à gauche }\end{array}$ & $\begin{array}{l}\text { A } \triangle \text { P CEY A [ ] AMACIAC } \\
\text { MHT NE ПP П ; } \\
\text { à l'exergue : ET C } \Theta\end{array}$ & R 407, no 520 \\
\hline & $206 / 207$ & $\begin{array}{l}\text { bûcher à un étage sur lequel se trouve un aigle } \\
\text { éployé de face, la tête à gauche tenant une } \\
\text { couronne dans son bec; à gauche, un arbre; } \\
\text { sur le bûcher, objets indistincts (branches ?) à } \\
\text { gauche et à droite de l'aigle (?) }\end{array}$ & $\begin{array}{l}\text { A } \triangle \text { P CEY ANT AMACIAC } \\
\text { MH NE ПP П ; } \\
\text { à l'exergue : ET C } \Theta\end{array}$ & R 408, no 521 \\
\hline & $206 / 207$ & $\begin{array}{l}\text { bûcher à un étage sur lequel se trouve un aigle } \\
\text { éployé de face, la tête à droite tenant une } \\
\text { couronne dans son bec; à gauche, un arbre }\end{array}$ & $\begin{array}{l}\text { A } \triangle \mathrm{P} \text { CEY A [ ] ; } \\
\text { à l'exergue : ET C } \Theta\end{array}$ & R 410, no 523 \\
\hline & $206 / 207$ & $\begin{array}{l}\text { bûcher à un étage sur lequel se trouve un aigle } \\
\text { éployé de face, la tête à gauche, tenant une } \\
\text { couronne dans son bec; à gauche, un arbre }\end{array}$ & $\begin{array}{l}\mathrm{A} \triangle \mathrm{P} \mathrm{CE}[\mathrm{]} \text { NE } \Pi \mathrm{P}, \\
\text { avec des variantes ; } \\
\text { à l'exergue : ET C } \Theta\end{array}$ & $\begin{array}{l}\text { R } 409, \text { no } 522 \\
\text { R } 411, \text { no }^{\circ} 524\end{array}$ \\
\hline & 206/207 & $\begin{array}{l}\text { bûcher à un étage sur lequel se trouve un aigle } \\
\text { éployé de face, la tête à gauche tenant une } \\
\text { couronne dans son bec; à gauche, un arbre; } \\
\text { une étoile dans le champ }\end{array}$ & $\begin{array}{l}\text { A } \triangle \text { P CEY ANT AMACIAC } \\
\text { MHT NE ПP } \Omega \text { ПON ; } \\
\text { dans le champ : ET C } \Theta\end{array}$ & R 412, no $^{\circ} 525$ \\
\hline & $206 / 207$ & $\begin{array}{l}\text { bûcher à un étage ; à gauche, un arbre ; sur } \\
\text { le bûcher un bœuf couché sur le dos avec à sa } \\
\text { gauche une branche ; au-dessus, un aigle éployé } \\
\text { de face, la tête à gauche, tenant une couronne } \\
\text { dans son bec ; une étoile dans le champ }\end{array}$ & $\begin{array}{l}\text { A } \triangle \text { P CEY ANT AMACIAC } \\
\text { MHT NE ПP П ; } \\
\text { à l'exergue : ET C } \Theta\end{array}$ & R 413, no 526 \\
\hline
\end{tabular}




\begin{tabular}{|c|c|c|c|c|}
\hline Empereur & Date & Description du type de revers & Légende & Références \\
\hline \multirow[t]{4}{*}{$\begin{array}{l}\text { Caracalla } \\
\text { (suite) }\end{array}$} & $206 / 207$ & $\begin{array}{l}\text { bûcher à un étage ; à gauche, un arbre ; sur le } \\
\text { bûcher une pyramide ; au-dessus Hélios dans } \\
\text { un quadrige formé de deux couples de chevaux } \\
\text { superposés dont on ne voit que la partie anté- } \\
\text { rieure de profil ; sur le bûcher, une branche à } \\
\text { gauche de la pyramide }\end{array}$ & $\begin{array}{l}\text { A } \triangle P \text { CEY ANT AMACIAC } \\
\text { MHT NE ПP П ; } \\
\text { à l'exergue : ET C } \Theta\end{array}$ & R 414, no 527 \\
\hline & $206 / 207$ & $\begin{array}{l}\text { bûcher à un étage ; à gauche, un arbre ; sur le } \\
\text { bûcher un aigle éployé de face, la tête à gauche; } \\
\text { au-dessus, Hélios dans un quadrige de face ; } \\
\text { à l'exergue, un bœuf couché sur le dos; une } \\
\text { étoile dans le champ à gauche }\end{array}$ & $\begin{array}{l}\text { A } \triangle \text { P CEY ANT AMACIAC } \\
\text { MHT NE ПP П ; } \\
\text { à l'exergue : ET C } \Theta\end{array}$ & $\begin{array}{l}\text { R } 247 \\
\text { nos } 529-530\end{array}$ \\
\hline & $206 / 207$ & $\begin{array}{l}\text { bûcher à un étage ; à gauche, un arbre ; sur le } \\
\text { bûcher une pyramide ; au-dessus, Hélios dans } \\
\text { un quadrige de face, le bras droit levé, un fouet } \\
\text { dans la main gauche ; une étoile dans le champ } \\
\text { à gauche }\end{array}$ & $\begin{array}{l}\text { A } \triangle P \text { CEY ANT AMACIAC } \\
\text { MH NE ПР П ; } \\
\text { à l'exergue : ET C } \Theta\end{array}$ & $\mathrm{R} 415, \mathrm{n}^{\circ} 528$ \\
\hline & $206 / 207$ & bûcher à un étage ; à gauche, un arbre & $\begin{array}{l}\text { A } \triangle \text { P CEY ANT AMACIAC } \\
\text { MHT NE ПP ПPO, } \\
\text { avec des variantes ; } \\
\text { dans le champ : ET C } \Theta\end{array}$ & $\begin{array}{l}\text { R } 417-418 \\
\text { nos } 533-534\end{array}$ \\
\hline \multirow[t]{9}{*}{ Géta César } & $205 / 206$ & $\begin{array}{l}\text { bûcher enflammé à deux étages; à gauche } \\
\text { un arbre ; sur le bûcher, des branches à gauche } \\
\text { et à droite des flammes }\end{array}$ & $\begin{array}{l}\text { A } \triangle P \text { CEY ANT AMACIAC } \\
\text { MHT NE ПP П ; } \\
\text { à l'exergue : ET CH }\end{array}$ & R 298 , no 549 \\
\hline & $205 / 206$ & $\begin{array}{l}\text { bûcher enflammé à deux étages ; } \\
\text { à gauche un arbre }\end{array}$ & $\begin{array}{l}\text { A } \triangle P \text { CEY ANT AMACIAC } \\
\text { MHT NE ПP П ; } \\
\text { à l'exergue : ET CH }\end{array}$ & R 202, no 551 \\
\hline & $205 / 206$ & $\begin{array}{l}\text { bûcher à un étage sur lequel se trouve un aigle } \\
\text { éployé de face, la tête à gauche tenant une } \\
\text { couronne dans son bec; à gauche, un arbre }\end{array}$ & $\begin{array}{l}\text { A } \triangle \text { P CEY ANT AMAC } \\
\text { MHT NE ПР ПO, } \\
\text { avec des variantes ; } \\
\text { à l'exergue : ET CH }\end{array}$ & $\begin{array}{l}\text { R } 217, \text { no } 554 \\
\text { R } 218, \text { no }^{\circ} 556 \\
\text { R } 427, \text { no } 552\end{array}$ \\
\hline & $205 / 206$ & $\begin{array}{l}\text { bûcher à un étage sur lequel se trouve un aigle } \\
\text { éployé de face, la tête à droite tenant une } \\
\text { couronne dans son bec; à gauche, un arbre }\end{array}$ & $\begin{array}{l}\text { A } \triangle \mathrm{P} \text { CEY AN [ ] ACIAC } \\
\text { MH NE ПPO ; } \\
\text { à l'exergue : ET CH }\end{array}$ & R 428, no 553 \\
\hline & $205 / 206$ & $\begin{array}{l}\text { bûcher à un étage sur lequel se trouve un aigle } \\
\text { éployé de face, la tête à droite tenant une } \\
\text { couronne dans son bec (?); à gauche, un arbre }\end{array}$ & $\begin{array}{l}\text { [ ]P CE AN A [ ] ; } \\
\text { à l'exergue : ET CH }\end{array}$ & R 429 , no 555 \\
\hline & $205 / 206$ & $\begin{array}{l}\text { bûcher à un étage ; à gauche, un arbre ; sur le } \\
\text { bûcher un bœuf couché sur le dos ; au-dessus, } \\
\text { un aigle éployé de face, la tête à gauche, tenant } \\
\text { une couronne dans son bec }\end{array}$ & $\begin{array}{l}\text { A } \triangle \text { P CEY ANT AMACIAC } \\
\text { MH NE ПР ПO ; } \\
\text { dans le champ : ET CH }\end{array}$ & R 431, no 557 \\
\hline & $205 / 206$ & $\begin{array}{l}\text { bûcher à un étage; à gauche, un arbre ; sur le } \\
\text { bûcher Hélios dans un quadrige de face, le bras } \\
\text { droit levé, un fouet dans la main gauche }\end{array}$ & $\begin{array}{l}\text { A } \triangle P \text { CEY ANT AMACI } \\
\text { MH NE ПР ПO ; } \\
\text { dans le champ : ET CH }\end{array}$ & R 223 , no 558 \\
\hline & $205 / 206$ & $\begin{array}{l}\text { bûcher à un étage ; à gauche, un arbre ; } \\
\text { sur le bûcher un quadrige de face }\end{array}$ & $\begin{array}{l}\text { A } \triangle \text { P CEY AN AMACI MH } \\
\text { NE ПR П MH ; } \\
\text { à l'exergue : ET CH }\end{array}$ & R 432, no 559 \\
\hline & $205 / 206$ & $\begin{array}{l}\text { bûcher à un étage ; à gauche, un arbre ; sur le } \\
\text { bûcher un aigle éployé de face, la tête à gauche; } \\
\text { au-dessus, Hélios dans un quadrige de face, le } \\
\text { bras droit levé, un fouet dans la main gauche }\end{array}$ & $\begin{array}{l}\text { A } \triangle \mathrm{P} \text { CEOY AN AMACIA } \\
\text { MH ; } \\
\text { à l'exergue : ET CH }\end{array}$ & R 433, no 560 \\
\hline
\end{tabular}


Anahita-Anaïtis/Zela

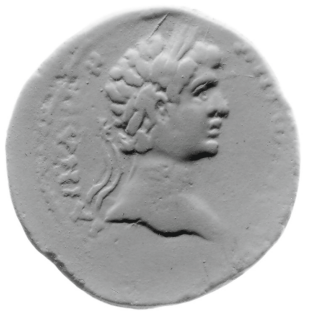

Figure 2
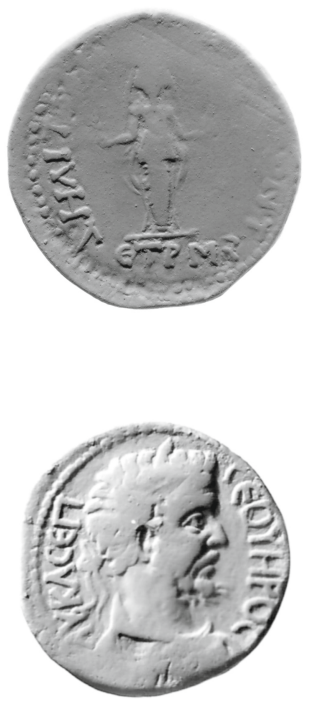

Figure 5

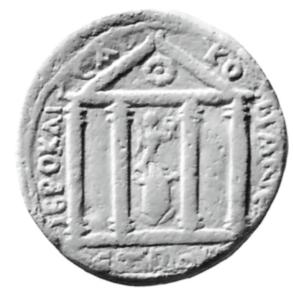

Mâ/Comana Pontica

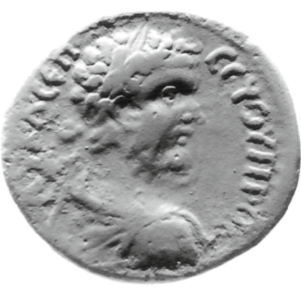

Figure 3
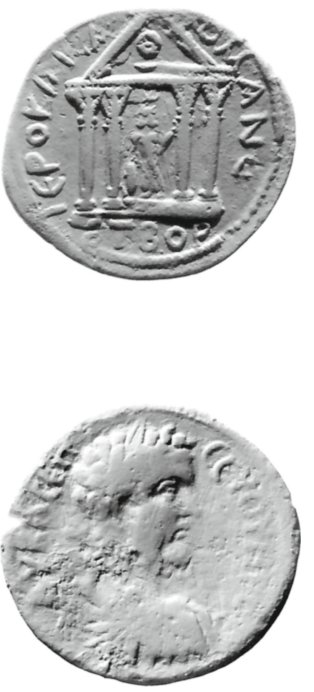

Figure 6

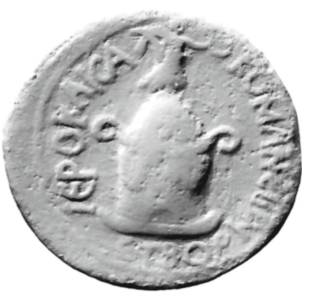

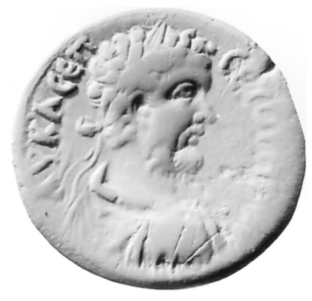

Figure 4

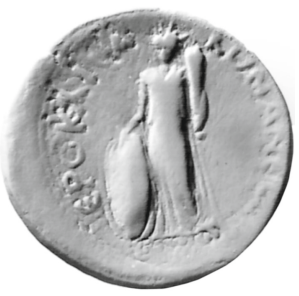

\section{Mèn/Neocaesarea}

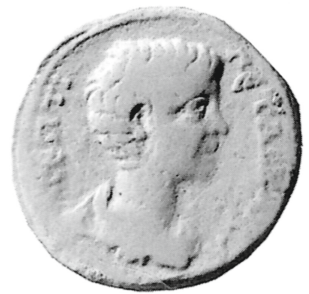

Figure 7

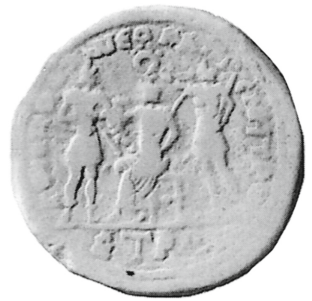

Figure 2 - Dalaison, Rémy, Amandry 2009, no 44a (Paris, FG 2000/428).

Figure 3 - AMANDRY, Rémy 1999, no 16a (Paris, FG 2001/50).

Figure 4 - AMANDRY, Rémy 1999, no 17a (Paris, FG 380).

Figure 5 - AMANDRY, RÉmy 1999, no 21a (Paris, Grand Duc 1354).

Figure 6 - AMANDRY, RÉmy 1999, no 36a (Paris, FG 381).

Figure 7 - ÇızMELı 2006, no 211a (Berlin, Löbbecke). 
Mithra/Trapezus

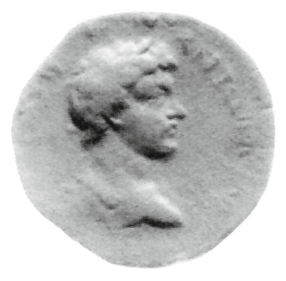

Figure 8
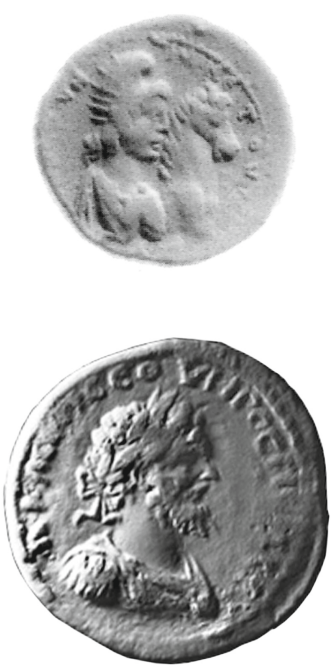

Figure 11

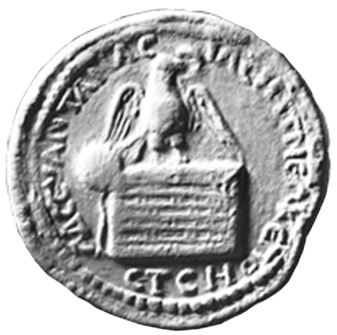

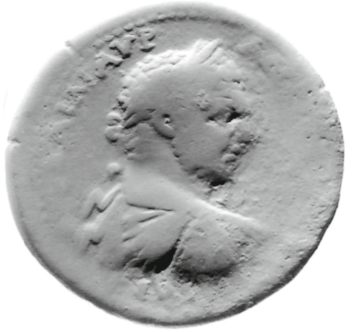

Figure 9
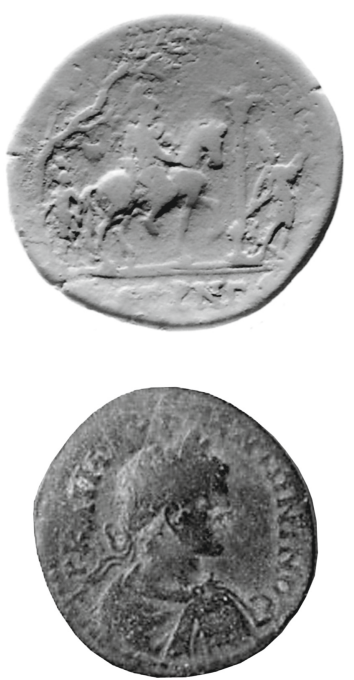

Figure 12

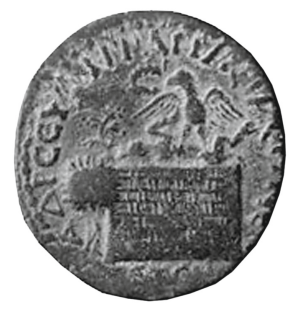

Zeus Stratios/Amaseia

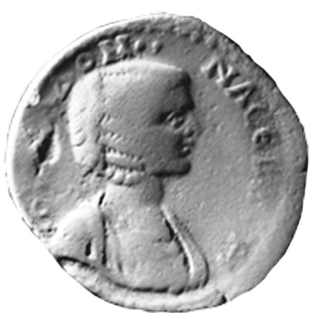

Figure 10
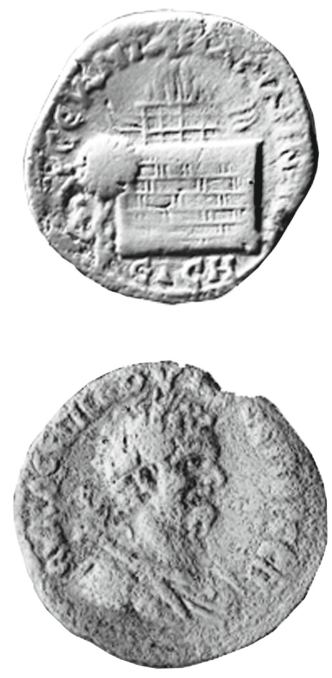

Figure 13

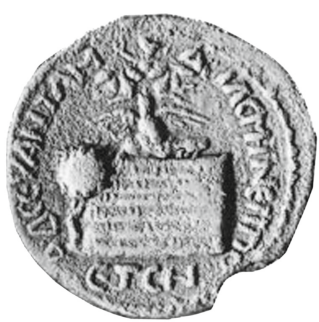

Figure 8 - WoJan 2006, no 41a (Bruxelles, II/30-622).

Figure 9 - WoJAn 2006, no 48a (Paris, FG 2001/53).

Figure 10 - Dalaison 2008, no 303a (Berlin, 1906 Löbbecke).

Figure 11 - DALAISON 2008, no 260a (Londres, BM 1897-1-2-4).

Figure 12 - Dalaison 2008, no 409a (Glasgow).

Figure 13 - DALAISON 2008, no 272a (Ankara, 11.92/59.91). 


\section{Bibliographie}

Amandry, Rémy 1998 : M. Amandry, B. Rémy, Pontica II. Les monnaies de l'atelier de Sebastopolis du Pont, (Varia Anatolica X), Paris, 1998.

Amandry, Rémy 1999 : M. Amandry, B. Rémy, Comana du Pont sous l'Empire romain. Étude historique et corpus monétaire, (Glaux 14), Milan, 1999.

Beard, North, Price 2006 : M. Beard, J. North, S. Price, Religions de Rome, Paris, 2006 (Cambridge, 1998 pour l'éd. anglaise).

BeCK 2004 : R. BECK, Beck on Mithraism, Aldertshot, 2004.

BECK 2006 : R. BECK, The Religion of the Mithras Cult in the Roman Empire, Oxford, 2006.

Belayche 2003 : N. Belayche, Tychè et la Tychè dans les cités de la Palestine romaine, Syria, 80, 2003, p. 111-138.

BelAyChe 2012 : N. Belayche, Introduction historiographique I. "L'homme de Mithra", dans Réédition critique de F. Cumont, Les Mystères de Mithra, N. Belayche, A. Mastrocinque (éd.), (Bibliotheca Cumontiana, Scripta maiora 3), Rome, 2012, p. XIII-LXVIII.

BERNADET 2009 : L. BERNADET, Les biens des temples dans l'Anatolie romaine : la question des "principautés sacerdotales", Archiv für Religionsgeschichte, 11, 2009, p. 63-87.

BERNADET 2011 : Laetitia $\rightleftharpoons$ NADET, Les signes divins au service du pouvoir sacerdotal en Anatolie ueménistique et romaine, dans La raison des signes. Présages, rites, destin dans les sociétés de la méditerranée ancienne, St. Georgoudi, R. Koch Piettre, Fr. Schmidt (éd.), (RGRW 174), Leyde, 2011, p. 293-317.

BRIANT 1996 : P. BRIANT, Histoire de l'Empire perse de Cyrus à Alexandre, Paris, 1996.

Byrne, Labarre 2006 : M.A. Byrne, G. Labarre, Nouvelles inscriptions d'Antioche de Pisidie d'après les Note-Books de W. M. Ramsay, (IK 67), Bonn, 2006.

Callataÿ 2003 : Fr. DE Callataÿ, Les Mithridate du Pont : un exemple périphérique de rapport entre cités et rois hellénistiques, dans Royaumes et cités hellénistiques de 323 à 55 av. J.-C., O. Picard, Fr. de Callataÿ, Fr. Duyrat, G. Corre, D. Prévot (éd.), Paris, 2003, p. 218-234.

Christol, Loriot 1986 : M. Christol, X. Loriot, Le Pontus et ses gouverneurs dans le second tiers du III siècle, dans Recherches épigraphiques : documents relatifs à l'histoire des institutions et de l'administration de l'Empire romain, B. Rémy (éd.), (Centre Jean Palerne, Mémoires 7), Saint-Étienne, 1986, p. 13-40.

Çızmeli 2006 : Z. Çızmeli, Le monnayage de Néocésarée et du koinon du Pont, (Glaux 17), Milan, 2006.

Cumont 1901 : Fr. Cumont, Le Zeus Stratios de Mithridate, Revue de l'histoire des religions, 43, 1901, p. 47-57.

Cumont et Cumont 1906 : Fr. et E. Cumont, Studia Pontica II. Voyage d'exploration archéologique dans le Pont et la petite Arménie, Bruxelles, 1906. 
Dalaison 2007 : J. Dalaison, L'atelier monétaire de Nicopolis en Arménie Mineure, dans Espaces et pouvoirs de l'Anatolie à la Gaule. Hommages à Bernard Rémy, J. Dalaison (éd.), Grenoble, 2007, p. 203-237.

Dalaison 2008 : J. Dalaison, L'atelier d'Amaseia du Pont. Recherches historiques et numismatiques, (Numismatica Anatolica 2), Bordeaux, 2008.

Dalaison 2010 : J. Dalaison, Le culte de Zeus Stratios à Amaseia du Pont sous l'Empire romain, dans La Norme religieuse dans l'Antiquité. Actes du colloque organisé les 14 et 15 décembre 2007 par les Universités de Lyon 2 et Lyon 3 , B. Cabouret, M.-O. Charles-Laforge (éd.), Lyon, 2010, p. 203-218.

Dalaison, Rémy, Amandry 2009 : J. Dalaison, B. Rémy, M. Amandry, Zéla sous l'Empire romain. Étude historique et corpus monétaire, (Numismatica Anatolica 4), Bordeaux, 2009.

DeBord 1982 : P. Debord, Aspects sociaux et économiques de la vie religieuse dans l'Anatolie gréco-romaine, (EPRO 88), Leyde, 1982.

DeININGER 1965 : J. DeININGER, Die Provinziallandtage der römischen Kaiserzeit von Augustus bis zum Ende des dritten Jahrhunderts $n$. Chr., (Vestigia, Beiträge zur alten Geschichte, Band 6), Munich, 1965.

Dignas 2002 : B. Dignas, Economy of the Sacred in Hellenistic and Roman Asia Minor, Oxford-New York, 2002.

FRENCH 1986 : D.H. FRENCH, Recent Epigraphic Research in Pontus, Epigraphica Anatolica, 8, 1986, p. 71-82.

Harl 1987 : K. W. Harl, Civic Coins and Civic Politics in the Roman East. AD 180-275, Berkeley-Los Angeles-Londres, 1987.

LABARRE 2009 : G. LABARRE, Les origines et la diffusion du culte de Men, dans L'Asie Mineure dans l'Antiquité : échanges, populations et territoires. Regards actuels sur une péninsule, H. Bru, Fr. Kirbihler, St. Lebreton (éd.), Rennes, 2009, p. 389-414.

Labarre 2010 : G. LABARre, Le dieu Mèn et son sanctuaire à Antioche de Pisidie, Bruxelles, 2010.

Lane 1967-1968 : E.N. Lane, A Re-Study of the God Men. Part III: Conclusions, Berytus, 17, 1967-1968, p. 81-106.

LoRiot 2006 : X. LoRIot, Le culte impérial dans le Pont sous le Haut-Empire, dans Pouvoir et religion dans le monde romain. En hommage à Jean-Pierre Martin, A. Vigourd, X. Loriot, A. Bérenger-Badel, B. Klein (éd.), Paris, 2006, p. 521-540.

LORIOT 2011 : X. LORIOT, La province de Pont-Bithynie sous le Haut-Empire : assise territoriale et administration, dans Fragments d'empire, fragments de mémoire. Pouvoirs et identités dans le monde romain impérial. IIe s. av.n.è.vie s. de n.è., St. Benoist, A. Daguet-Gagey, Chr. Hoët-van Cauwenberghe (éd.), Lille, 2011, p. 257-286.

Marek 2003 : Chr. Marek, Pontus et Bithynia. Die römischen Provinzen im norden Kleinasiens, Mainz, 2003.

MareK 2010 : Chr. MareK, Geschichte Kleinasiens in der Antike, Munich, 2010. 
Michels 2009 : Chr. Michels, Kulturtransfer und monarchischer «Philhellenismus». Bithynien, Pontos und Kappadokien in hellenistischer Zeit, Göttingen, 2009.

Mitchell 1993 : St. Mitchell, Anatolia. Land, Men, and Gods in Asia Minor. Volume II : The Rise of the Church, Oxford, 1993.

Mitchell 2007a : St. Mitchell, Iranian Names and the Presence of Persians in the Religious Sanctuaries of Asia Minor, Proceedings of the British Academy, 148, 2007, p. 151-171.

Mitchell 2007b : St. Mitchell, Römische Macht im frühkaiserzeitlichen Ankara. Verwaltung oder Herrschaft?, dans Herrschen und Verwalten. Der Alltag der römischen Administration in der Hoherkaiserzeit, R. Haensch, J. Heinrich (éd.), Köln-Vienne, 2007, p. 366-377.

Nony 1971 : D. Nony, Une monnaie de Césarée de Cappadoce au nom de Statorius Secundus, légat d'Hadrien, BSFN, 26, 1971, p. 37-38.

Olshausen 1990 : E. Olshausen, Götter, Heroen und ihre Kulte in Pontos - ein erster Bericht, ANRW, II, 18-3, Berlin-New York, p. 1865-1906.

Proeva 1992 : N. Proeva, Ma, LIMC, VI, 1992, p. 330-331.

RÉMY 1986 : B. RÉMY, L'évolution administrative de l'Anatolie aux trois premiers siècles de notre ère, CEROR, Lyon, 1986.

RÉMY 1989 : B. RÉMY, Les carrières sénatoriales dans les provinces romaines d'Anatolie au Haut-Empire (31 avant J.-C.-284 après J.-C.) - Pont-Bithynie, Galatie, Cappadoce, Lycie-Pamphylie et Cilicie, IFEA, Istanbul, 1989.

SAPRYKIN 2009 : S. J. SAPRYKIN, The Religion and Cults of the Pontic Kingdom, dans Mithridates VI and the Pontic Kingdom, J.M. Højte (éd.), (Black Sea Studies 9), 2009, p. 249-275.

SARTRE 2003 : M. SARTre, L'Anatolie hellénistique de l'Égée au Caucase, Paris, 2003.

Schultz 1989 : H.-D. Schultz, Megalopolis-Sebasteia, dans Kraay-M $\phi$ rkholm Essays. Numismatic Studies in Memory of C. M. Kraay and O. Mфrkholm, G. Le Rider, K. Jenkins, N. Waggoner, U. Westermark (éd.), Louvain-la-Neuve, 1989, p. 259-266.

SöKMEN 2009 : E. SöKMEN, Characteristics of the Temple States in Pontus, dans Mithridates VI and the Pontic Kingdom, J.M. Højte (éd.), (Black Sea Studies 9), 2009, p. 277-287.

Sydenham 1933 : A A. Sydenham, The Coinage of Caesarea in Cappadocia, Londres, 1933.

TeIXIDOR 1981 : J. TeIXIDOR, Anaeitis, LIMC, I, 1981, p. 754-756.

TURCAN 1992 : R. TuRCAN, Les cultes orientaux dans le monde romain, Paris, 1992.

Turcan 1993 : R. TurCan, Mithra et le mithriacisme, Paris, 1993.

VollKommer 1992a : R. Vollkommer, Men, LIMC, VI, 1992, p. 462-472.

VollKommer 1992b : R. Vollkommer, Mithras, LIMC, VI, 1992, p. 583-626.

Waddington, Babelon, Reinach 1908 : W. H. Waddington, E. Babelon, Th. ReInACH, Recueil général des monnaies grecques d'Asie Mineure. I, 2. Bithynie (jusqu'à Juliopolis), Paris, 1908. 
Waddington, Babelon, Reinach 1912 : W.H. Waddington, E. Babelon, Th. ReInACH, Recueil général des monnaies grecques d'Asie Mineure. I, 4. Prusa, Prusias, Tius, Paris, 1912.

Waddington, Babelon et Reinach 1925 : W.H. Waddington, E. Babelon, Th. ReInACH, Recueil général des monnaies grecques d'Asie Mineure. I, 1. Pont et Paphlagonie, Paris, 1925 (2e éd.).

Wojan 2003 : Fr. Wojan, Kérasonte du Pont sous l'Empire romain. Étude historique et corpus monétaire, $R N, 159,2003$, p. 257-290.

Wojan 2006 : Fr. Wojan, Trapézonte du Pont sous l'Empire romain : étude historique et corpus monétaire, $R N, 162,2006$, p. 181-229. 